TRANSACTIONS OF THE

AMERICAN MATHEMATICAL SOCIETY

Volume 357, Number 5 , Pages 1931-1952

S 0002-9947(04)03519-6

Article electronically published on July 22, 2004

\title{
ASSOCIATIVITY OF CROSSED PRODUCTS BY PARTIAL ACTIONS, ENVELOPING ACTIONS AND PARTIAL REPRESENTATIONS
}

\author{
M. DOKUCHAEV AND R. EXEL
}

\begin{abstract}
Given a partial action $\alpha$ of a group $G$ on an associative algebra $\mathcal{A}$, we consider the crossed product $\mathcal{A} \rtimes_{\alpha} G$. Using the algebras of multipliers, we generalize a result of Exel (1997) on the associativity of $\mathcal{A} \rtimes_{\alpha} G$ obtained in the context of $C^{*}$-algebras. In particular, we prove that $\mathcal{A} \rtimes_{\alpha} G$ is associative, provided that $\mathcal{A}$ is semiprime. We also give a criterion for the existence of a global extension of a given partial action on an algebra, and use crossed products to study relations between partial actions of groups on algebras and partial representations. As an application we endow partial group algebras with a crossed product structure.
\end{abstract}

\section{INTRODUCTION}

Partial actions of groups appeared independently in various areas of mathematics, in particular, in the theory of operator algebras as a powerful tool in their study (see [8, 9], [10, [15, [17]). In the most general setting of partial actions on abstract sets the definition is as follows:

Definition 1.1. Let $G$ be a group with identity element 1 and $\mathcal{X}$ be a set. A partial action $\alpha$ of $G$ on $\mathcal{X}$ is a collection of subsets $\mathcal{D}_{g} \subseteq \mathcal{X}(g \in G)$ and bijections $\alpha_{g}: \mathcal{D}_{g^{-1}} \rightarrow \mathcal{D}_{g}$ such that

(i) $\mathcal{D}_{1}=\mathcal{X}$ and $\alpha_{1}$ is the identity map of $\mathcal{X}$

(ii) $\mathcal{D}_{(g h)^{-1}} \supseteq \alpha_{h}^{-1}\left(\mathcal{D}_{h} \cap \mathcal{D}_{g^{-1}}\right)$;

(iii) $\alpha_{g} \circ \alpha_{h}(x)=\alpha_{g h}(x)$ for each $x \in \alpha_{h}^{-1}\left(\mathcal{D}_{h} \cap \mathcal{D}_{g^{-1}}\right)$.

Note that conditions (ii) and (iii) mean that the function $\alpha_{g h}$ is an extension of the function $\alpha_{g} \circ \alpha_{h}$. Moreover, it is easily seen that (ii) can be replaced by a "stronger looking" condition: $\alpha_{h}^{-1}\left(\mathcal{D}_{h} \cap \mathcal{D}_{g^{-1}}\right)=\mathcal{D}_{h^{-1}} \cap \mathcal{D}_{h^{-1} g^{-1}}$. Indeed, it obviously follows from (ii) that $\alpha_{h}^{-1}\left(\mathcal{D}_{h} \cap \mathcal{D}_{g^{-1}}\right) \subseteq \mathcal{D}_{h^{-1}} \cap \mathcal{D}_{h^{-1} g^{-1}}$. Replacing $h$ by $h^{-1}$ and $g$ by $g h$, we have $\alpha_{h^{-1}}^{-1}\left(\mathcal{D}_{h^{-1}} \cap \mathcal{D}_{h^{-1} g^{-1}}\right) \subseteq \mathcal{D}_{h} \cap \mathcal{D}_{g^{-1}}$, and consequently, $\alpha_{h^{-1}}\left(\mathcal{D}_{h} \cap \mathcal{D}_{g^{-1}}\right) \supseteq \mathcal{D}_{h^{-1}} \cap \mathcal{D}_{h^{-1} g^{-1}}$. By (iii) $\alpha_{h^{-1}}=\alpha_{h}^{-1}$, and we obtain the desired

Received by the editors February 19, 2003 and, in revised form, September 26, 2003.

2000 Mathematics Subject Classification. Primary 16S99; Secondary 16S10, 16S34, 16S35, 16W22, 16W50, 20C07, 20L05.

Key words and phrases. Partial action, crossed product, partial representation, partial group ring, grading, groupoid.

This work was partially supported by CNPq of Brazil (Proc. 301115/95-8, Proc. 303968/85-0). 
equality. Thus, the conditions (i)-(iii) are equivalent to the following:

(i) $\mathcal{D}_{1}=\mathcal{X}$ and $\alpha_{1}$ is the identity map of $\mathcal{X}$;

(ii') $\alpha_{g}\left(\mathcal{D}_{g^{-1}} \cap \mathcal{D}_{h}\right)=\mathcal{D}_{g} \cap \mathcal{D}_{g h}$;

(iii') $\alpha_{g}\left(\alpha_{h}(x)\right)=\alpha_{g h}(x)$, for all $x \in \mathcal{D}_{h^{-1}} \cap \mathcal{D}_{(g h)^{-1}}$.

Let $S(G)$ be the universal semigroup generated by the symbols $\{[g]: g \in G\}$ subject to relations:

a) $\left[g^{-1}\right][g][h]=\left[g^{-1}\right][g h]$;

b) $[g][h]\left[h^{-1}\right]=[g h]\left[h^{-1}\right] \quad(g, h \in G)$;

c) $[1]=1$,

where 1 denotes also the identity element of $S(G)$. Then $S(G)$ is an inverse semigroup and the partial actions of $G$ on $\mathcal{X}$ are in one-to-one correspondence with (ordinary) actions of $S(G)$ on $\mathcal{X}[10$.

In what follows, by an algebra we shall mean an associative non-unital (i.e. not necessarily unital) algebra. In order to define a partial action $\alpha$ of a group $G$ on a $K$-algebra $\mathcal{A}$ we suppose in Definition 1.1 that each $\mathcal{D}_{g}(g \in G)$ is an ideal of $\mathcal{A}$ and that every map $\alpha_{g}: \mathcal{D}_{g^{-1}} \rightarrow \mathcal{D}_{g}$ is an isomorphism of algebras.

Together with the notion of partial actions, a generalization of the concept of crossed product appeared in the theory of operator algebras (see [8, 9], 11, [15]). For simplicity we assume that the twisting is trivial, so we give the definition in the context of corresponding skew group rings.

Definition 1.2. Given a partial action $\alpha$ of a group $G$ on an algebra $\mathcal{A}$, the skew group ring $\mathcal{A} \rtimes_{\alpha} G$ corresponding to $\alpha$ is the set of all finite formal sums $\left\{\sum_{g \in G} a_{g} \delta_{g}: a_{g} \in \mathcal{D}_{g}\right\}$, where $\delta_{g}$ are symbols. Addition is defined in the obvious way, and multiplication is determined by $\left(a_{g} \delta_{g}\right) \cdot\left(b_{h} \delta_{h}\right)=\alpha_{g}\left(\alpha_{g^{-1}}\left(a_{g}\right) b_{h}\right) \delta_{g h}$.

Obviously, $\mathcal{A} \ni a \mapsto a \delta_{1} \in \mathcal{A} \rtimes_{\alpha} G$ is an embedding which permits us to identify $\mathcal{A}$ with $\mathcal{A} \delta_{1}$. The first question which naturally arises is whether or not $\mathcal{A} \rtimes_{\alpha} G$ is associative. The associativity of this construction was proved in [8] in the context of $C^{*}$-algebras roughly ten years ago. Since the $C^{*}$-algebraic proof employed very special properties of $C^{*}$-algebras (the existence of approximate units), the associativity question remained open since then for partial action on general algebras.

One of our goals is to prove that $\mathcal{A} \rtimes_{\alpha} G$ is always associative if $\mathcal{A}$ is semiprime (Corollary 3.4). Recall that a unital algebra $\mathcal{A}$ is called semiprime if $\mathcal{A}$ has no nonzero nilpotent ideal (for other equivalent definitions see [18, Theorem 2.6.17]). More generally, given a partial action of $G$ on a unital algebra $\mathcal{A}$, the skew group ring $\mathcal{A} \rtimes_{\alpha} G$ is associative provided that each ideal $\mathcal{D}_{g}$ is idempotent or non-degenerate (Corollary 3.2). We say that an ideal $\mathcal{I}$ of $\mathcal{A}$ is non-degenerate if for every non-zero element $a \in \mathcal{I}$ there exists $b \in \mathcal{I}$ such that either $a b \neq 0$ or $b a \neq 0$. It is easily proved that a unital algebra $\mathcal{A}$ is semiprime if and only if each non-zero ideal of $\mathcal{A}$ is non-degenerate (see Proposition 2.6).

Note that in a $C^{*}$-algebraic context the ideals $\mathcal{D}_{g}$ are supposed to be closed by definition, and it is known that each closed ideal in a $C^{*}$-algebra is an idempotent ideal (see [13, Theorem V.9.2]).

We also show that in general $\mathcal{A} \rtimes_{\alpha} G$ is not associative (see Section 3). In Section 4 we give a complete answer for the problem of the existence of a "global extension" (called enveloping action) for a given partial action on a unital algebra. More precisely, an enveloping action exists if and only if each ideal $\mathcal{D}_{g}$ is generated by a central idempotent (see Theorem 4.5). We introduce the notion of enveloping action with a certain minimality condition in order to guarantee its uniqueness up 
to equivalence (see Section 4 for the definitions). We also prove that if $(\mathcal{B}, \beta)$ is an enveloping action for a partial action $(\mathcal{A}, \alpha)$ such that $\mathcal{A}$ and $\mathcal{B}$ are unital algebras, then the corresponding skew group rings, namely $\mathcal{A} \rtimes_{\alpha} G$ and $\mathcal{B} \rtimes_{\beta} G$, are Morita equivalent.

Another relevant concept which appeared in the theory of operator algebras is the notion of partial representation of a group on a Hilbert space, introduced independently in [10] and [17. Partial representations underlie important algebras generated by partial isometries. Among the most interesting cases are the CuntzKrieger algebras [4, investigated in [11] and [12] from the point of view of partial representations. The purely algebraic study of partial representations began in [5] and was continued in [6] (see also [7]). Similarly to the case of (usual) representations of groups, there exists an algebra $K_{\mathrm{par}}(G)$, called the partial group algebra of $G$ over $K$, which governs the partial representations of $G$ (see [5]). It is exactly the semigroup algebra $K S(G)$. It turns out that $K_{\text {par }}(G)$ keeps much more information about the structure of $G$ than does $K G$ (see [5]), and this makes them especially interesting with respect to the classical isomorphism problem intensively investigated for group rings (see [19]).

In the final Section 6 we use crossed products to relate partial actions with partial representations. This leads to a one-to-one correspondence (see Theorem 6.11) when working with so-called "elementary" partial representations, which are structural blocks of the irreducible partial representations (see [6]). This permits us to look at matrix algebras as crossed products by partial actions (see Corollary 6.12), covering in this way a substantial part of elementary gradings (see Corollary 6.15). Moreover, starting with the partial representation $G \ni g \mapsto[g] \in K_{\mathrm{par}}(G)$, we endow $K_{\text {par }}(G)$ with a crossed product structure (see Theorem [6.9).

\section{The ALGebra of MUltipliers}

Let $K$ be a field, $\mathcal{A}$ an associative $K$-algebra with unity element and $\mathcal{I}$ an ideal of $\mathcal{A}$. Take an element $x \in \mathcal{A}$ and consider the left and right multiplications of $\mathcal{I}$ by $x: L_{x}: \mathcal{I} \ni a \mapsto x a \in \mathcal{I}, \quad R_{x}: \mathcal{I} \ni a \mapsto a x \in \mathcal{I}$. Then $L=L_{x}$ and $R=R_{x}$ are linear transformations of $\mathcal{I}$ such that the following properties are satisfied for all $a, b \in \mathcal{I}$ :

(i) $L(a b)=L(a) b$;

(ii) $R(a b)=a R(b)$;

(iii) $R(a) b=a L(b)$.

These properties are obvious consequences of the associativity of $\mathcal{A}$.

Definition 2.1. The algebra of multipliers (see, e.g., 14, 3.12.2]) of an algebra $\mathcal{I}$ is the set $M(\mathcal{I})$ of all ordered pairs $(L, R)$, where $L$ and $R$ are linear transformations of $\mathcal{I}$ which satisfy the properties (i)-(iii). For $(L, R),\left(L^{\prime}, R^{\prime}\right) \in M(\mathcal{I})$ and $\alpha \in K$ the operations are given by

$$
\begin{gathered}
\alpha(L, R)=(\alpha L, \alpha R), \\
(L, R)+\left(L^{\prime}, R^{\prime}\right)=\left(L+L^{\prime}, R+R^{\prime}\right), \\
(L, R)\left(L^{\prime}, R^{\prime}\right)=\left(L \circ L^{\prime}, R^{\prime} \circ R\right) .
\end{gathered}
$$

We say that $L$ is a left multiplier and $R$ is a right multiplier of $\mathcal{I}$.

It is immediately verified that $M(\mathcal{I})$ is an associative algebra with unity element $\left(L_{1}, R_{1}\right)$, where $L_{1}$ and $R_{1}$ are identity maps (which in the case of an ideal $\mathcal{I}$ in 
a unital algebra $\mathcal{A}$ can be considered as multiplications by the unity element of $\mathcal{A}$ from left and right, respectively). Define the map $\phi: \mathcal{I} \rightarrow M(\mathcal{I})$ by putting $\phi(x)=\left(L_{x}, R_{x}\right), x \in \mathcal{I}$. This is a homomorphism of algebras, since it is $K$-linear and, moreover, $L_{x y}=L_{x} \circ L_{y}, R_{x y}=R_{y} \circ R_{x}$, which gives

$$
\phi(x y)=\left(L_{x} \circ L_{y}, R_{y} \circ R_{x}\right)=\phi(x) \phi(y) .
$$

Definition 2.2. We shall say that an algebra $\mathcal{I}$ is non-degenerate if the map $\phi: \mathcal{I} \rightarrow M(\mathcal{I})$ mentioned above is injective.

In general the kernel of $\phi$ is the intersection of the left annihilator of $\mathcal{I}$ in $\mathcal{I}$ with its right annihilator in $\mathcal{I}$. Therefore $\mathcal{I}$ is non-degenerate if and only if for every non-zero element $a \in \mathcal{I}$ there exists $b \in \mathcal{I}$ such that either $a b \neq 0$ or $b a \neq 0$.

More generally, if $\mathcal{I}$ is an ideal in an algebra $\mathcal{A}$, then one may consider the homomorphism $\psi: \mathcal{A} \ni a \mapsto\left(L_{a}, R_{a}\right) \in M(\mathcal{I})$, whose kernel is the intersection of the left annihilator of $\mathcal{I}$ in $\mathcal{A}$ with its right annihilator in $\mathcal{A}$.

Proposition 2.3. The following statements hold:

(i) $\phi(\mathcal{I})$ is an ideal of $M(\mathcal{I})$.

(ii) $\phi: \mathcal{I} \rightarrow \mathcal{M}(\mathcal{I})$ is an isomorphism if and only if $\mathcal{I}$ is a unital algebra.

Proof. (i) Take $x \in \mathcal{I}$ and let $(L, R)$ be an arbitrary element of $M(\mathcal{I})$. Then $\left(L_{x}, R_{x}\right)(L, R)=\left(L_{x} \circ L, R \circ R_{x}\right)$, and for $a \in \mathcal{I}$ we have $L_{x}(L(a))=x L(a)=$ $R(x) a=L_{R(x)}(a)$. Moreover, $R\left(R_{x}(a)\right)=R(a x)=a R(x)=R_{R(x)}(a)$. Hence, $\left(L_{x}, R_{x}\right)(L, R)=\left(L_{R(x)}, R_{R(x)}\right) \in \phi(\mathcal{I})$, as $R(x) \in \mathcal{I}$. Similarly, $(L, R)\left(L_{x}, R_{x}\right)=$ $\left(L_{L(x)}, R_{L(x)}\right) \in \phi(\mathcal{I})$.

(ii) The "only if" part is trivial. For the "if" part we have that $\phi(1) \in \phi(\mathcal{I})$ is the identity element of $M(\mathcal{I})$ and, consequently, $\phi(\mathcal{I})=M(\mathcal{I})$. Obviously, $\phi$ is injective in this case, and thus $\mathcal{I} \cong M(\mathcal{I})$.

Let $\mathcal{I}$ be any (preferably non-unital) algebra. Given $(L, R)$ and $\left(L^{\prime}, R^{\prime}\right)$ in $\mathcal{M}(\mathcal{I})$, we shall be concerned with the validity of the formula

$$
R^{\prime} \circ L=L \circ R^{\prime} \text {. }
$$

If $x$ and $x^{\prime}$ belong to an algebra which contains $\mathcal{I}$ as an ideal and $(L, R)=$ $\left(L_{x}, R_{x}\right)$ and $\left(L^{\prime}, R^{\prime}\right)=\left(L_{x^{\prime}}, R_{x^{\prime}}\right)$, this formula will clearly hold as a consequence of associativity. However this is not always the case: for a very drastic counterexample one could take $\mathcal{I}$ to be any vector space equipped with the trivial multiplication

$$
x y \equiv 0, \quad \forall x, y \in \mathcal{I} .
$$

Any pair $(L, R)$ of linear operators on $\mathcal{I}$ would constitute a multiplier of $\mathcal{I}$, and one would clearly not expect (II) to hold!

Definition 2.4. An algebra $\mathcal{I}$ is said to be $(L, R)$-associative if, given any two multipliers $(L, R)$ and $\left(L^{\prime}, R^{\prime}\right)$ in $\mathcal{M}(\mathcal{I})$, one has that $R^{\prime} \circ L=L \circ R^{\prime}$.

The following result lists two sufficient conditions for (L,R)-associativity.

Proposition 2.5. The algebra $\mathcal{I}$ is $(L, R)$-associative whenever any one of the following conditions hold:

(i) $\mathcal{I}$ is non-degenerate, or

(ii) $\mathcal{I}$ is idempotent. 
Proof. Let $(L, R),\left(L^{\prime}, R^{\prime}\right) \in \mathcal{M}(\mathcal{I})$. Given $a, b \in \mathcal{I}$, we have that

$$
R\left(L^{\prime}(a)\right) b=L^{\prime}(a) L(b)=L^{\prime}(a L(b))=L^{\prime}(R(a) b)=L^{\prime}(R(a)) b .
$$

This shows that $R\left(L^{\prime}(a)\right)-L^{\prime}(R(a))$ lies in the left annihilator of $\mathcal{I}$. By a similar calculation one shows that $R\left(L^{\prime}(a)\right)-L^{\prime}(R(a))$ lies in the right annihilator of $\mathcal{I}$ as well. Therefore, under assumption $(i)$, one must have that $R\left(L^{\prime}(a)\right)=L^{\prime}(R(a))$, for all $a$ in $\mathcal{I}$.

Next suppose we are given $a_{1}, a_{2} \in \mathcal{I}$. Letting $a=a_{1} a_{2}$, notice that

$$
\begin{gathered}
R\left(L^{\prime}(a)\right)=R\left(L^{\prime}\left(a_{1} a_{2}\right)\right)=R\left(L^{\prime}\left(a_{1}\right) a_{2}\right)=L^{\prime}\left(a_{1}\right) R\left(a_{2}\right) \\
=L^{\prime}\left(a_{1} R\left(a_{2}\right)\right)=L^{\prime}\left(R\left(a_{1} a_{2}\right)\right)=L^{\prime}(R(a)) .
\end{gathered}
$$

Assuming (ii), we have that every element of $\mathcal{I}$ is a sum of terms of the form $a_{1} a_{2}$, whence the conclusion.

In the next section we will be considering partial actions such that all of the ideals $D_{g}$ are assumed to be $(\mathrm{L}, \mathrm{R})$-associative. It is therefore useful to have a means of deciding when all ideals of a certain algebra possess this property. The following easy result goes in that direction.

Proposition 2.6. Let $\mathcal{A}$ be a unital algebra. The following are equivalent:

(i) Every non-zero ideal of $\mathcal{A}$ is non-degenerate.

(ii) Every non-zero ideal of $\mathcal{A}$ is either idempotent or non-degenerate.

(iii) Every non-zero ideal of $\mathcal{A}$ is right-non-degenerate (we say that $\mathcal{I}$ is rightnon-degenerate if for every non-zero element $a \in \mathcal{I}$ one has that aI $\neq\{0\}$ ).

(iv) Every non-zero ideal of $\mathcal{A}$ is left-non-degenerate (defined in a similar way with $\mathcal{I} a \neq\{0\})$.

(v) $\mathcal{A}$ is semiprime.

In this case every ideal of $\mathcal{A}$ is $(L, R)$-associative.

Proof. In the following implications the ones marked with a star are self-evident:

$$
\text { (i) } \stackrel{*}{\Rightarrow} \text { (ii) } \Rightarrow \text { (v) } \Rightarrow \text { (iii) } \stackrel{*}{\Rightarrow} \text { (i). }
$$

Moreover, (iv) can be substituted for (iii), by symmetry, so we really only need to worry about (ii) $\Rightarrow$ (v) $\Rightarrow$ (iii).

If $\mathcal{A}$ has a non-zero nilpotent ideal, then there is a non-zero ideal $\mathcal{I}$ in $\mathcal{A}$ whose square is zero. Then $\mathcal{I}$ is neither idempotent nor non-degenerate, which shows (ii) $\Rightarrow(\mathrm{v})$.

Assuming (v), and arguing by contradiction, let $\mathcal{I}$ be an ideal possessing a nonzero element $a$ such that $a \mathcal{I}=\{0\}$. Then the ideal generated by $a$, namely $\mathcal{J}=$ $\mathcal{A} a \mathcal{A}$, is non-zero since we are assuming $\mathcal{A}$ to be unital. However,

$$
\mathcal{J}^{2}=\mathcal{A} a(\mathcal{A} \mathcal{A} a) \mathcal{A} \subseteq \mathcal{A} a \mathcal{I} \mathcal{A}=\{0\},
$$

violating (v).

Let $\pi: \mathcal{I} \rightarrow \mathcal{J}$ be an isomorphism of $K$-algebras. Then it is easy to see that for $(L, R) \in M(\mathcal{I})$ the pair $\left(\pi \circ L \circ \pi^{-1}, \pi \circ R \circ \pi^{-1}\right)$ is an element of $M(\mathcal{J})$, and we obviously have the following:

Proposition 2.7. The map $\bar{\pi}: M(\mathcal{I}) \rightarrow M(\mathcal{J})$, defined by

$$
\bar{\pi}(L, R)=\left(\pi \circ L \circ \pi^{-1}, \pi \circ R \circ \pi^{-1}\right),
$$

is an isomorphism of $K$-algebras. 


\section{The ASsociativity QUeSTION}

We are now ready to present an answer to the associativity question:

Theorem 3.1. If $\mathcal{A}$ is an algebra and $\alpha$ is a partial action of a group $G$ on $\mathcal{A}$ such that each $\mathcal{D}_{g}(g \in G)$ is $(L, R)$-associative, then the skew group ring $\mathcal{A} \rtimes_{\alpha} G$ is associative.

Proof. Obviously, $\mathcal{A} \rtimes_{\alpha} G$ is associative if and only if

$$
\left(a \delta_{h} b \delta_{g}\right) c \delta_{f}=a \delta_{h}\left(b \delta_{g} c \delta_{f}\right)
$$

for arbitrary $h, g, f \in G$ and $a \in \mathcal{D}_{h}, b \in \mathcal{D}_{g}, c \in \mathcal{D}_{f}$. First we compute the left hand side of the above equality. We have

$$
\left(a \delta_{h} b \delta_{g}\right) c \delta_{f}=\alpha_{h}\left(\alpha_{h^{-1}}(a) b\right) \delta_{h g} c \delta_{f}=\alpha_{h g}\left\{\alpha_{h g}^{-1}\left[\alpha_{h}\left(\alpha_{h^{-1}}(a) b\right)\right] c\right\} \delta_{h g f} .
$$

We see that $\alpha_{h^{-1}}(a) b \in \mathcal{D}_{h^{-1}} \cap \mathcal{D}_{g}$ implies

$$
\alpha_{h}\left(\alpha_{h^{-1}}(a) b\right) \in \alpha_{h}\left(\mathcal{D}_{h^{-1}} \cap \mathcal{D}_{g}\right)=\mathcal{D}_{h} \cap \mathcal{D}_{h g} .
$$

It follows that

$$
\alpha_{h g}^{-1}\left[\alpha_{h}\left(\alpha_{h^{-1}}(a) b\right)\right]=\alpha_{g^{-1}}\left(\alpha_{h^{-1}}\left[\alpha_{h}\left(\alpha_{h^{-1}}(a) b\right)\right]\right)=\alpha_{g^{-1}}\left(\alpha_{h^{-1}}(a) b\right) .
$$

Since this element belongs to $\mathcal{D}_{g^{-1}} \cap \mathcal{D}_{g^{-1} h^{-1}}$, we can also split $\alpha_{h g}$, which gives

$$
\left(a \delta_{h} b \delta_{g}\right) c \delta_{f}=\alpha_{h}\left[\alpha_{g}\left\{\alpha_{g^{-1}}\left(\alpha_{h^{-1}}(a) b\right) c\right\}\right] \delta_{h g f} .
$$

Comparing with

$$
a \delta_{h}\left(b \delta_{g} c \delta_{f}\right)=a \delta_{h} \alpha_{g}\left(\alpha_{g^{-1}}(b) c\right) \delta_{g f}=\alpha_{h}\left[\alpha_{h^{-1}}(a) \alpha_{g}\left(\alpha_{g^{-1}}(b) c\right)\right] \delta_{h g f},
$$

and applying $\alpha_{h^{-1}}$, we obtain that (2) holds if and only if the equality

$$
\alpha_{g}\left\{\alpha_{g^{-1}}\left(\alpha_{h^{-1}}(a) b\right) c\right\}=\alpha_{h^{-1}}(a) \alpha_{g}\left(\alpha_{g^{-1}}(b) c\right)
$$

is verified for all $a \in \mathcal{D}_{h}, b \in \mathcal{D}_{g}, c \in \mathcal{D}_{f}$. Because $\alpha_{h^{-1}}: \mathcal{D}_{h} \longrightarrow \mathcal{D}_{h^{-1}}$ is an isomorphism, $\alpha_{h^{-1}}(a)$ runs over $\mathcal{D}_{h^{-1}}$ and, consequently, the above condition is equivalent to the following:

$$
\alpha_{g}\left\{\alpha_{g^{-1}}(a b) c\right\}=a \alpha_{g}\left(\alpha_{g^{-1}}(b) c\right)
$$

for every $a \in \mathcal{D}_{h^{-1}}, b \in \mathcal{D}_{g}, c \in \mathcal{D}_{f}$. If $h=f=1$, then $\mathcal{D}_{h}=\mathcal{D}_{f}=\mathcal{A}$, and thus $\mathcal{A} \rtimes_{\alpha} G$ is associative if and only if (3) holds for arbitrary $g \in G, a, c \in \mathcal{A}$ and $b \in \mathcal{D}_{g}$. It is equivalent to say that

$$
\left(\alpha_{g} \circ R_{c} \circ \alpha_{g^{-1}}\right) \circ L_{a}=L_{a} \circ\left(\alpha_{g} \circ R_{c} \circ \alpha_{g^{-1}}\right)
$$

is valid on $\mathcal{D}_{g}$ for every $g \in G$ and all $a, c \in \mathcal{A}$.

Consider $R_{c}$ as a right multiplier of $\mathcal{D}_{g^{-1}}$ and $L_{a}$ as a left multiplier of $\mathcal{D}_{g}$. By Proposition 2.7 we have that $\alpha_{g} \circ R_{c} \circ \alpha_{g^{-1}}$ is a right multiplier of $\mathcal{D}_{g}$. Hence if $\mathcal{D}_{g}$ is $(\mathrm{L}, \mathrm{R})$-associative, then (4) holds.

Corollary 3.2. If $\alpha$ is a partial action of a group $G$ on an algebra $\mathcal{A}$ such that each $\mathcal{D}_{g}(g \in G)$ is either idempotent or non-degenerate, then the skew group ring $\mathcal{A} \rtimes_{\alpha} G$ is associative.

Proof. This directly follows from Proposition 2.5 and Theorem 3.1 . 
For ease of reference it is useful to introduce the following terminology:

Definition 3.3. We say that an algebra $\mathcal{A}$ is strongly associative if for any group $G$ and an arbitrary partial action $\alpha$ of $G$ on $\mathcal{A}$ the skew group $\operatorname{ring} \mathcal{A} \rtimes_{\alpha} G$ is associative.

As an immediate consequence of Proposition 2.6 and Corollary 3.2 we have

Corollary 3.4. A semiprime algebra is strongly associative.

The following is an easy example which shows that $\mathcal{A} \rtimes_{\alpha} G$ is not associative in general.

Example 3.5. Let $\mathcal{A}$ be a four-dimensional $K$-vector space with basis $\{1, t, u, v\}$. Define the multiplication on $\mathcal{A}$ by setting $u^{2}=v^{2}=u v=v u=t u=u t=t^{2}=$ $0, t v=v t=u$ and $1 a=a 1=a$ for each $a \in \mathcal{A}$. Then $\mathcal{A}$ is an associative $K$-algebra with unity. Let $G=\left\langle g: g^{2}=1\right\rangle$, and $\mathcal{I}$ the ideal of $\mathcal{A}$ generated by $v$ (it is the subspace generated by $u$ and $v$ ). Consider the partial action $\alpha$ of $G$ on $\mathcal{A}$ given by $\mathcal{D}_{g}=\mathcal{I}, \alpha_{g}: u \mapsto v, v \mapsto u$ (by definition $\mathcal{D}_{1}=\mathcal{A}$ and $\alpha_{1}$ is the identity map of $\mathcal{A})$. Then the skew group $\operatorname{ring} \mathcal{A} \rtimes_{\alpha} G$ is not associative. More precisely, taking $x=t \delta_{1}+u \delta_{g}$ we have that $(x x) x=0$ and $x(x x)=u \delta_{g}$, so that $\mathcal{A} \rtimes_{\alpha} G$ does not even have associative powers.

An important class of non-semiprime algebras is formed by the group algebras $K G$ of finite groups $G$ with char $K$ dividing the order of $G$ (for more general information, see [16. Theorems 2.12 and 2.13]). It is easy to check that if $\operatorname{char} K=2$, then the algebra of the above example is isomorphic to the group algebra of the Klein four-group over $K$. On the other hand, it can be verified that the group algebra of the cyclic group of order 2 over a field of characteristic 2 is strongly associative. Thus it would be interesting to characterize the strongly associative group algebras. Another classical example of non-semiprime algebras is given by the algebra $T(n, K)$ of upper triangular $n \times n$-matrices over $K$.

Proposition 3.6. The algebra $\mathcal{A}=T(n, K)$ is strongly associative if and only if $n \leq 2$.

Proof. If $n=1$, then $\mathcal{A}=K$ is obviously strongly associative. If $n=2$, then the only non-idempotent ideal of $\mathcal{A}$ is its Jacobson radical $\mathcal{R}(\mathcal{A})$, which is onedimensional over $K$. So all multipliers of $\mathcal{R}(\mathcal{A})$ commute and hence $\mathcal{A}$ is (L,R)associative.

Suppose that $n \geq 3$ and let $G=\langle g\rangle$ be the infinite cyclic group. Denote by $e_{i, j}$ the elementary $n \times n$ matrix whose unique non-zero entry equals 1 and is placed at the intersection of the $i$-th row and $j$-th column. Take $\mathcal{D}_{g^{-1}}=e_{1, n-1} K \oplus e_{1, n} K$, $\mathcal{D}_{g}=e_{1, n} K \oplus e_{2, n} K$ and $\mathcal{D}_{g^{m}}=e_{1, n} K$ for each $m$ with $|m| \geq 2$. Define $\alpha_{g}$ : $\mathcal{D}_{g^{-1}} \rightarrow \mathcal{D}_{g}$ by $\alpha_{g}\left(x e_{1, n-1}+y e_{1, n}\right)=y e_{1, n}+x e_{2, n}(x, y \in K)$, and for $m \geq 2$ let $\alpha_{g^{m}}: \mathcal{D}_{g^{-m}}=\mathcal{D}_{g^{m}} \rightarrow \mathcal{D}_{g^{m}}$ be the identity map. An easy verification shows that we have defined a partial action $\alpha$ of $G$ on $\mathcal{A}$. We see that

$$
\left(e_{1,1} \delta_{1} \cdot e_{2, n} \delta_{g}\right) e_{n-1, n} \delta_{1}=\left(e_{1,1} \cdot e_{2, n} \delta_{g}\right) e_{n-1, n} \delta_{1}=0,
$$

as $e_{1,1} \cdot e_{2, n}=0$. On the other hand,

$$
\begin{aligned}
& e_{1,1} \delta_{1}\left(e_{2, n} \delta_{g} \cdot e_{n-1, n} \delta_{1}\right)=e_{1,1} \delta_{1}\left(\alpha_{g}\left(\alpha_{g^{-1}}\left(e_{2, n}\right) e_{n-1, n}\right) \delta_{g}\right) \\
& =e_{1,1} \delta_{1}\left(\alpha_{g}\left(e_{1, n-1} \cdot e_{n-1, n}\right) \delta_{g}\right)=e_{1,1} \delta_{1} \cdot \alpha_{g}\left(e_{1, n}\right) \delta_{g}=e_{1,1} \delta_{1} e_{1, n} \delta_{g} \\
& =\left(e_{1,1} \cdot e_{1, n}\right) \delta_{g}=e_{1, n} \delta_{g} \neq 0,
\end{aligned}
$$

so that $\mathcal{A} \rtimes_{\alpha} G$ is non-associative. 


\section{Enveloping aCtions}

Natural examples of partial actions can be obtained by restricting (global) actions to not necessarily invariant subsets (ideals in our case). More precisely, suppose that a group $G$ acts on an algebra $\mathcal{B}$ by automorphisms $\beta_{g}: \mathcal{B} \rightarrow \mathcal{B}$ and let $\mathcal{A}$ be an ideal of $\mathcal{B}$. Set $\mathcal{D}_{g}=\mathcal{A} \cap \beta_{g}(\mathcal{A})$ and let $\alpha_{g}$ be the restriction of $\beta_{g}$ to $\mathcal{D}_{g^{-1}}$. Then it is easily verified that we have a partial action $\alpha=\left\{\alpha_{g}: \mathcal{D}_{g^{-1}} \rightarrow \mathcal{D}_{g}: g \in G\right\}$ of $G$ on $\mathcal{A}$. We shall say that $\alpha$ is a restriction of $\beta$ to $\mathcal{A}$. We want to know circumstances under which a given partial action can be obtained "up to equivalence" as the restriction of a (global) action. If $\mathcal{B}_{1}$ is the subalgebra of $\mathcal{B}$ generated by $\bigcup_{g \in G} \beta_{g}(\mathcal{A})$, it may happen that $\mathcal{B} \neq \mathcal{B}_{1}$ and, since $\mathcal{B}_{1}$ is invariant with respect to $\beta$, we see that $\alpha$ can be obtained as a restriction of an action of $G$ to $\mathcal{B}_{1}$ which is a proper subalgebra of $\mathcal{B}$. Thus it is reasonable to require that $\mathcal{B}=\mathcal{B}_{1}$, and in this case we say that $\alpha$ is an admissible restriction of $\beta$. The notion of the equivalence of partial actions is defined as follows:

Definition 4.1. We say that a partial action $\alpha=\left\{\alpha_{g}: \mathcal{D}_{g^{-1}} \rightarrow \mathcal{D}_{g}: g \in G\right\}$ of a group $G$ on an algebra $\mathcal{A}$ is equivalent to the partial action $\alpha^{\prime}=\left\{\alpha_{g}^{\prime}\right.$ : $\left.\mathcal{D}^{\prime}{ }_{g}^{-1} \rightarrow \mathcal{D}^{\prime}{ }_{g}: g \in G\right\}$ of $G$ on an algebra $\mathcal{A}^{\prime}$ if there exists an algebra isomorphism $\varphi: \mathcal{A} \rightarrow \mathcal{A}^{\prime}$ such that for every $g \in G$ the following two conditions hold:

(i) $\varphi\left(\mathcal{D}_{g}\right)=\mathcal{D}_{g}^{\prime}$;

(ii) $\alpha_{g}^{\prime} \circ \varphi(x)=\varphi \circ \alpha_{g}(x)$ for all $x \in \mathcal{D}_{g^{-1}}$.

We shall deal with enveloping actions:

Definition 4.2. An action $\beta$ of a group $G$ on an algebra $\mathcal{B}$ is said to be an enveloping action for the partial action $\alpha$ of $G$ on an algebra $\mathcal{A}$ if $\alpha$ is equivalent to an admissible restriction of $\beta$ to an ideal of $\mathcal{B}$.

In other words, $\beta$ is an enveloping action for $\alpha$ if there exists an algebra isomorphism $\varphi$ of $\mathcal{A}$ onto an ideal of $\mathcal{B}$ such that for all $g \in G$ the following three properties are satisfied:

$\left(\mathrm{i}^{\prime}\right) \varphi\left(\mathcal{D}_{g}\right)=\varphi(\mathcal{A}) \cap \beta_{g}(\varphi(\mathcal{A})) ;$

$\left(\right.$ ii $\left.^{\prime}\right) \varphi \circ \alpha_{g}(x)=\beta_{g} \circ \varphi(x)$ for each $x \in \mathcal{D}_{g^{-1}}$;

(iii') $\mathcal{B}$ is generated by $\bigcup_{g \in G} \beta_{g}(\varphi(\mathcal{A}))$.

Thus a general problem is to decide whether or not a given partial action possesses an enveloping action.

With respect to the results of Section 3 we observe the following:

Proposition 4.3. If $\beta$ is an action of a group $G$ on an algebra $\mathcal{B}$, which is enveloping for the partial action $\alpha$ of $G$ on an algebra $\mathcal{A}$, then the skew group ring $\mathcal{A} \rtimes_{\alpha} G$ has an embedding into $\mathcal{B} \rtimes_{\beta} G$. In particular, $\mathcal{A} \rtimes_{\alpha} G$ is associative.

Proof. Obvious.

Thus it follows from Example [3.5 (or Proposition [3.6) that not every partial action admits an enveloping action. We need the following easy fact:

Lemma 4.4. Suppose that $\mathcal{A}$ is an algebra which is a (not necessarily direct) sum of a finite number of ideals, each of which is a unital algebra. Then $\mathcal{A}$ is a unital algebra.

Proof. By induction on the number of summands it is enough to consider the case with two ideals: $\mathcal{A}=\mathcal{I}+\mathcal{J}$. Let $1_{\mathcal{I}}$ and $1_{\mathcal{J}}$ be the unity elements of $\mathcal{I}$ and $\mathcal{J}$ 
respectively. Then $1_{\mathcal{I}}$ and $1_{\mathcal{J}}$ are central idempotents of $\mathcal{A}$ and $1_{\mathcal{I}}+1_{\mathcal{J}}-1_{\mathcal{I}} \cdot 1_{\mathcal{J}}$ is the unity of $\mathcal{A}$.

The following result is inspired by F. Abadie's thesis [1] (see also [2]):

Theorem 4.5. Let $\mathcal{A}$ be a unital algebra. Then a partial action $\alpha$ of a group $G$ on $\mathcal{A}$ admits an enveloping action $\beta$ if and only if each ideal $\mathcal{D}_{g}(g \in G)$ is a unital algebra. Moreover, $\beta$, if it exists, is unique up to equivalence.

Proof. The "only if" part is trivial, because if $\beta$ exists and $\varphi: \mathcal{A} \rightarrow \mathcal{B}$ is the monomorphism giving the equivalence, then $\varphi\left(\mathcal{D}_{g}\right)=\varphi(\mathcal{A}) \cap \beta_{g}(\varphi(\mathcal{A}))$ is clearly a unital algebra for each $g \in G$.

For the "if" part we suppose that each ideal $\mathcal{D}_{g}(g \in G)$ is a unital algebra. This means that for every $g \in G$ there exists a central idempotent $1_{g}$ of $\mathcal{A}$ such that $\mathcal{D}_{g}=1_{g} \mathcal{A}$.

Let $\mathcal{F}=\mathcal{F}(G, \mathcal{A})$ be the Cartesian product of the copies of $\mathcal{A}$ indexed by the elements of $G$, that is, the algebra of all functions of $G$ into $\mathcal{A}$. For convenience of notation $f(g)$ will be also written as $\left.f\right|_{g}(f \in \mathcal{F}, g \in G)$.

For $g \in G$ and $f \in \mathcal{F}$ define $\beta_{g}(f) \in \mathcal{F}$ by the formula:

$$
\left.\beta_{g}(f)\right|_{h}=f\left(g^{-1} h\right), h \in G .
$$

It is easily verified that $f \mapsto \beta_{g}(f)$ defines an automorphism $\beta_{g}$ of $\mathcal{F}$, and hence $\beta=\left\{\beta_{g}: \mathcal{F} \rightarrow \mathcal{F}: g \in G\right\}$ is an action of $G$ on $\mathcal{F}$.

It is easy to see that for each $g, h \in G$ the idempotent $1_{g} 1_{h}$ is the unity element of the algebra $\mathcal{D}_{g} \cap \mathcal{D}_{h}$, which means that $\mathcal{D}_{g} \cap \mathcal{D}_{h}=1_{g} 1_{h} \mathcal{A}$. Because $\alpha$ is a partial action, the equality $\alpha_{g}\left(\mathcal{D}_{g^{-1}} \cap \mathcal{D}_{h}\right)=\mathcal{D}_{g} \cap \mathcal{D}_{g h}$ obviously implies

$$
\alpha_{g}\left(1_{g^{-1}} 1_{h}\right)=1_{g} 1_{g h} .
$$

For any $a \in \mathcal{A}$ the element $a 1_{g}$ belongs to $\mathcal{D}_{g}$, and the formula

$$
\left.\varphi(a)\right|_{g}=\alpha_{g^{-1}}\left(a 1_{g}\right), g \in G,
$$

defines a monomorphism $\varphi: \mathcal{A} \rightarrow \mathcal{F}$.

Let $\mathcal{B}$ be the subalgebra of $\mathcal{F}$ generated by $\bigcup_{g \in G} \beta_{g}(\varphi(\mathcal{A}))(g \in G)$. Our purpose is to show that the restriction of $\beta$ to $\mathcal{B}$ is an enveloping action for $\alpha$. We denote this restriction by the same symbol $\beta$. We start by checking property (ii') of Definition 4.2 .

For $g, h \in G$ and $a \in \mathcal{D}_{g^{-1}}$ we have $\left.\beta_{g}(\varphi(a))\right|_{h}=\left.\varphi(a)\right|_{g^{-1} h}=\alpha_{h^{-1} g}\left(a 1_{g^{-1} h}\right)$ and $\left.\varphi\left(\alpha_{g}(a)\right)\right|_{h}=\alpha_{h^{-1}}\left(\alpha_{g}(a) 1_{h}\right)$. Thus (ii') is satisfied if and only if the following equality holds for all $g, h \in G$ and every $a \in \mathcal{D}_{g^{-1}}$ :

$$
\alpha_{h^{-1} g}\left(a 1_{g^{-1} h}\right)=\alpha_{h^{-1}}\left(\alpha_{g}(a) 1_{h}\right) .
$$

Observing that $a \cdot 1_{g^{-1} h} \in \mathcal{D}_{g^{-1}} \cap \mathcal{D}_{g^{-1} h}$, we can split $\alpha_{h^{-1} g}$ in the left-hand side, and using (5) we obtain

$$
\begin{aligned}
& \alpha_{h^{-1} g}\left(a 1_{g^{-1} h}\right)=\alpha_{h^{-1}}\left(\alpha_{g}\left(a 1_{g^{-1} h}\right)\right)=\alpha_{h^{-1}}\left(\alpha_{g}\left(a 1_{g^{-1}} 1_{g^{-1} h}\right)\right) \\
& =\alpha_{h^{-1}}\left(\alpha_{g}(a) \alpha_{g}\left(1_{g^{-1}} 1_{g^{-1} h}\right)\right)=\alpha_{h^{-1}}\left(\alpha_{g}(a) 1_{g} 1_{h}\right)=\alpha_{h^{-1}}\left(\alpha_{g}(a) 1_{h}\right),
\end{aligned}
$$

as $1_{g}$ is the unity of $\mathcal{D}_{g}$.

Next we show that

$$
\varphi\left(\mathcal{D}_{g}\right)=\varphi(\mathcal{A}) \cap \beta_{g}(\varphi(\mathcal{A}))
$$


for all $g \in G$. An element from the right-hand side can be written as $\varphi(a)=\beta_{g}(\varphi(b))$ for some $a, b \in \mathcal{A}$. Then for each $h \in G$ the equality $\left.\varphi(a)\right|_{h}=\left.\beta_{g}(\varphi(b))\right|_{h}$ means that

$$
\alpha_{h^{-1}}\left(a 1_{h}\right)=\left.\varphi(b)\right|_{g^{-1} h}=\alpha_{h^{-1} g}\left(b 1_{g^{-1} h}\right) .
$$

Taking $h=1$, this gives $a=\alpha_{g}\left(b 1_{g^{-1}}\right) \in \mathcal{D}_{g}$ and, consequently, $\varphi\left(\mathcal{D}_{g}\right) \supseteq \varphi(\mathcal{A}) \cap$ $\beta_{g}(\varphi(\mathcal{A}))$. For the reverse inclusion, given an element $a \in \mathcal{D}_{g}$, we need to find $b \in \mathcal{A}$ so that (8) holds. For $b=\alpha_{g^{-1}}(a)$ the right-hand side of (8) is $\alpha_{h^{-1} g}\left(\alpha_{g^{-1}}(a) 1_{g^{-1} h}\right)$, which is equal to the left-hand side of (8) in view of (6). Hence (7) follows and condition $\left(\mathrm{i}^{\prime}\right)$ is also satisfied.

In order to show that $\beta$ is an enveloping action for $\alpha$ it remains to prove that $\varphi(\mathcal{A})$ is an ideal of $\mathcal{B}$. To see this it is enough to check that $\beta_{g}(\varphi(a)) \cdot \varphi(b), \varphi(b) \cdot \beta_{g}(\varphi(a)) \in$ $\varphi(\mathcal{A})$ for all $g \in G$ and $a, b \in \mathcal{A}$. For $h \in G$, using ([6), we have

$$
\begin{aligned}
& \left.\left.\beta_{g}(\varphi(a))\right|_{h} \cdot \varphi(b)\right|_{h}=\left.\left.\varphi(a)\right|_{g^{-1} h} \cdot \varphi(b)\right|_{h}=\alpha_{h^{-1} g}\left(a 1_{g^{-1} h}\right) \cdot \alpha_{h^{-1}}\left(b 1_{h}\right) \\
& =\alpha_{h^{-1}}\left(\alpha_{g}\left(a 1_{g^{-1}}\right) 1_{h}\right) \cdot \alpha_{h^{-1}}\left(b 1_{h}\right)=\alpha_{h^{-1}}\left(\alpha_{g}\left(a 1_{g^{-1}}\right) b 1_{h}\right)=\left.\varphi\left(\alpha_{g}\left(a 1_{g^{-1}}\right) b\right)\right|_{h} .
\end{aligned}
$$

Thus $\beta_{g}(\varphi(a)) \cdot \varphi(b)=\varphi\left(\alpha_{g}\left(a 1_{g^{-1}}\right) b\right) \in \varphi(\mathcal{A})$ and similarly $\varphi(b) \cdot \beta_{g}(\varphi(a))=$ $\varphi\left(b \alpha_{g}\left(a 1_{g^{-1}}\right)\right) \in \varphi(\mathcal{A})$, as desired.

Now we shall prove the uniqueness of the enveloping action. Suppose that $\beta^{\prime}$ is another action of $G$ on an algebra $\mathcal{B}^{\prime}$, which is enveloping for $\alpha$. Let $\varphi^{\prime}$ be the corresponding embedding of $\mathcal{A}$ into $\mathcal{B}^{\prime}$. The admissibility incorporated in Definition 4.2 means that $\mathcal{B}^{\prime}$ is the sum of the ideals $\beta_{g}^{\prime}\left(\varphi^{\prime}(\mathcal{A})\right), g \in G$. Thus an element of $\mathcal{B}^{\prime}$ can be written as a finite sum $\sum_{i} \beta_{g_{i}}^{\prime}\left(\varphi^{\prime}\left(a_{i}\right)\right)$ with $g_{i} \in G$ and $a_{i} \in \mathcal{A}$. Define a map $\phi: \mathcal{B}^{\prime} \rightarrow \mathcal{B}$ by $\beta_{g}^{\prime}\left(\varphi^{\prime}(a)\right) \mapsto \beta_{g}(\varphi(a)), g \in G, a \in \mathcal{A}$. We have to show, of course, that $\phi$ is well defined. Suppose that $\sum_{i=1}^{s} \beta_{g_{i}}^{\prime}\left(\varphi^{\prime}\left(a_{i}\right)\right)=0$. We want to be sure that $\sum_{i=1}^{s} \beta_{g_{i}}\left(\varphi\left(a_{i}\right)\right)=0$.

For all $h \in G$ and $a \in \mathcal{A}$ we have $\sum_{i} \beta_{g_{i}}^{\prime}\left(\varphi^{\prime}\left(a_{i}\right)\right) \beta_{h}^{\prime}\left(\varphi^{\prime}(a)\right)=0$, and, applying $\beta_{h^{-1}}^{\prime}$, we obtain $\sum_{i} \beta_{h^{-1} g_{i}}^{\prime}\left(\varphi^{\prime}\left(a_{i}\right)\right) \varphi^{\prime}(a)=0$. Since $\varphi^{\prime}(\mathcal{A})$ is an ideal in $\mathcal{B}^{\prime}$, the element $\beta_{h^{-1} g_{i}}^{\prime}\left(\varphi^{\prime}\left(a_{i}\right)\right) \varphi^{\prime}(a)$ is contained in the algebra $\beta_{h^{-1} g_{i}}^{\prime}\left(\varphi^{\prime}(\mathcal{A})\right) \cap \varphi^{\prime}(\mathcal{A})=$ $\varphi^{\prime}\left(\mathcal{D}_{h^{-1} g_{i}}\right)=\varphi^{\prime}\left(\mathcal{A} 1_{h^{-1} g_{i}}\right)=\varphi^{\prime}(\mathcal{A}) \varphi^{\prime}\left(1_{h^{-1} g_{i}}\right)$, whose unity element is $\varphi^{\prime}\left(1_{h^{-1} g_{i}}\right)$. Therefore, using (ii'),

$$
\begin{aligned}
& \beta_{h^{-1} g_{i}}^{\prime}\left(\varphi^{\prime}\left(a_{i}\right)\right) \varphi^{\prime}(a)=\beta_{h^{-1} g_{i}}^{\prime}\left(\varphi^{\prime}\left(a_{i}\right)\right) \varphi^{\prime}\left(1_{h^{-1} g_{i}}\right) \varphi^{\prime}(a) \\
& =\beta_{h^{-1} g_{i}}^{\prime}\left(\varphi^{\prime}\left(a_{i}\right)\right) \varphi^{\prime} \circ \alpha_{h^{-1} g_{i}}\left(1_{g_{i}-1}\right) \varphi^{\prime}(a) \\
& =\beta_{h^{-1} g_{i}}^{\prime}\left(\varphi^{\prime}\left(a_{i}\right)\right) \beta_{h^{-1} g_{i}}^{\prime} \circ \varphi^{\prime}\left(1_{g_{i}-1}\right) \varphi^{\prime}(a) \\
& =\beta_{h^{-1} g_{i}}^{\prime} \circ \varphi^{\prime}\left(a_{i} 1_{g_{i}-1}\right) \varphi^{\prime}(a) \\
& =\varphi^{\prime} \circ \alpha_{h^{-1} g_{i}}\left(a_{i} 1_{g_{i}-1}\right) \varphi^{\prime}(a)=\varphi^{\prime}\left(\alpha_{h^{-1} g_{i}}\left(a_{i} 1_{g_{i}-1}\right) a\right) .
\end{aligned}
$$

In a similar fashion we see that $\beta_{h^{-1} g_{i}}\left(\varphi\left(a_{i}\right)\right) \varphi(a)=\varphi\left(\alpha_{h^{-1} g_{i}}\left(a_{i} 1_{g_{i}-1 h}\right) a\right)$. Thus,

$$
0=\sum_{i=1}^{s} \beta_{h^{-1} g_{i}}^{\prime}\left(\varphi^{\prime}\left(a_{i}\right)\right) \varphi^{\prime}(a)=\sum_{i=1}^{s} \varphi^{\prime}\left(\alpha_{h^{-1} g_{i}}\left(a_{i} 1_{g_{i}-1}\right) a\right),
$$

which implies $\sum_{i=1}^{s} \alpha_{h^{-1} g_{i}}\left(a_{i} 1_{g_{i}-1} h\right)=0$. Hence

$$
0=\sum_{i=1}^{s} \varphi\left(\alpha_{h^{-1} g_{i}}\left(a_{i} 1_{g_{i}-1}\right) a\right)=\sum_{i=1}^{s} \beta_{h^{-1} g_{i}}\left(\varphi\left(a_{i}\right)\right) \varphi(a),
$$


and applying $\beta_{h}$ we obtain

$$
\sum_{i=1}^{s} \beta_{g_{i}}\left(\varphi\left(a_{i}\right)\right) \beta_{h}(\varphi(a))=0,
$$

for all $a \in \mathcal{A}$. Therefore, the element $\sum_{i=1}^{s} \beta_{g_{i}}\left(\varphi\left(a_{i}\right)\right)$ annihilates each $\beta_{h}(\varphi(\mathcal{A}))$. Let $\mathcal{B}_{1}$ be the algebra generated by $\bigcup_{i=1}^{s} \beta_{g_{i}}(\varphi(\mathcal{A}))$. Then $\sum_{i=1}^{s} \beta_{g_{i}}\left(\varphi\left(a_{i}\right)\right) \in \mathcal{B}_{1}$, and by Lemma $4.4 \mathcal{B}_{1}$ possesses a unity element, say $1_{\mathcal{B}_{1}}$. Then

$$
\sum_{i=1}^{s} \beta_{g_{i}}\left(\varphi\left(a_{i}\right)\right)=\sum_{i=1}^{s} \beta_{g_{i}}\left(\varphi\left(a_{i}\right)\right) \cdot 1_{\mathcal{B}_{1}}=0,
$$

so that $\sum_{i=1}^{s} \beta_{g_{i}}^{\prime}\left(\varphi^{\prime}\left(a_{i}\right)\right)=0$ implies $\sum_{i=1}^{s} \beta_{g_{i}}\left(\varphi\left(a_{i}\right)\right)=0$ and $\phi: \mathcal{B}^{\prime} \rightarrow \mathcal{B}$ is a well-defined homomorphism of algebras.

By symmetry, $\beta_{g}(\varphi(a)) \mapsto \beta_{g}^{\prime}\left(\varphi^{\prime}(a)\right), g \in G, a \in \mathcal{A}$, also determines a welldefined map $\phi^{\prime}: \mathcal{B} \rightarrow \mathcal{B}^{\prime}$. Obviously, $\phi^{\prime} \circ \phi=\phi \circ \phi^{\prime}=1$ and, consequently, $\phi$ is an isomorphism of algebras. It is easily seen that for all $g \in G$ one has $\beta_{g} \circ \phi=\phi \circ \beta_{g}^{\prime}$, and this yields that $\beta^{\prime}$ is equivalent to $\beta$.

\section{Morita equivalence}

Recall from [18, Section 4.1] that a Morita context is a six-tuple $\left(R, R^{\prime}, M, M^{\prime}\right.$, $\left.\tau, \tau^{\prime}\right)$, where:

(a) $R$ and $R^{\prime}$ are rings,

(b) $M$ is an $R$ - $R^{\prime}$-bimodule,

(c) $M^{\prime}$ is an $R^{\prime}$ - $R$-bimodule,

(d) $\tau: M \otimes_{R^{\prime}} M^{\prime} \rightarrow R$ is a bimodule map,

(e) $\tau^{\prime}: M^{\prime} \otimes_{R} M \rightarrow R^{\prime}$ is a bimodule map, such that

$$
\tau\left(x \otimes x^{\prime}\right) y=x \tau^{\prime}\left(x^{\prime} \otimes y\right), \quad \forall x, y \in M, x^{\prime} \in M^{\prime},
$$

and

$$
\tau^{\prime}\left(x^{\prime} \otimes x\right) y^{\prime}=x^{\prime} \tau\left(x \otimes y^{\prime}\right), \quad \forall x^{\prime}, y^{\prime} \in M^{\prime}, x \in M
$$

By Morita's fundamental results [18, Theorems 4.1.4 and 4.1.17], given a Morita context with $\tau$ and $\tau^{\prime}$ onto, the categories of $R$-modules and of $R^{\prime}$-modules are equivalent. In this case $R$ and $R^{\prime}$ are said to be Morita equivalent.

Let $\alpha$ be a partial action of a group $G$ on a unital algebra $\mathcal{A}$ and suppose that $(\beta, \mathcal{B})$ is an enveloping action for $(\alpha, \mathcal{A})$ such that $\mathcal{B}$ also has unity. It is our goal in this section to exhibit an explicit Morita context for the rings $R=\mathcal{A} \rtimes_{\alpha} G$ and $R^{\prime}=\mathcal{B} \rtimes_{\beta} G$, with $\tau$ and $\tau^{\prime}$ onto, hence proving these to be Morita equivalent. This should be seen as a purely algebraic counterpart to [2] Theorem 4.18].

Consider the linear subspaces $M, N \subseteq \mathcal{B} \rtimes_{\beta} G$ given by

$$
M=\left\{\sum_{g \in G} c_{g} \delta_{g}: c_{g} \in \mathcal{A} \text { for all } g\right\}
$$

and

$$
N=\left\{\sum_{g \in G} c_{g} \delta_{g}: c_{g} \in \beta_{g}(\mathcal{A}) \text { for all } g\right\} .
$$

Proposition 5.1. $M$ is a right ideal and $N$ is a left ideal of $\mathcal{B} \rtimes_{\beta} G$. 
Proof. Let $c \delta_{g}$ be in $M$, where $g \in G$ and $c \in \mathcal{A}$. If $h \in G$ and $b \in \mathcal{B}$, we have

$$
c \delta_{g} \cdot b \delta_{h}=c \beta_{g}(b) \delta_{g h} \in M,
$$

because $c \beta_{g}(b) \in \mathcal{A}$ since $\mathcal{A}$ is an ideal in $\mathcal{B}$. So $M$ is a right ideal in $\mathcal{B} \rtimes_{\beta} G$.

Next let $c \delta_{g}$ be in $N$, where $g \in G$ and $c=\beta_{g}\left(c^{\prime}\right)$ with $c^{\prime} \in \mathcal{A}$. If $h \in G$ and $b \in \mathcal{B}$, we have

$$
b \delta_{h} \cdot c \delta_{g}=b \beta_{h}(c) \delta_{h g}=b \beta_{h g}\left(c^{\prime}\right) \delta_{h g} \in N,
$$

because $\beta_{h g}(\mathcal{A})$ is an ideal in $\mathcal{B}$. So $N$ is a left ideal.

In our next result we will view $\mathcal{A} \rtimes_{\alpha} G$ as a subalgebra of $\mathcal{B} \rtimes_{\beta} G$ as in (4.3).

Proposition 5.2. $\left(\mathcal{A} \rtimes_{\alpha} G\right) M \subseteq M$ and $N\left(\mathcal{A} \rtimes_{\alpha} G\right) \subseteq N$, so that $M$ may be viewed as a left $\mathcal{A} \rtimes_{\alpha} G$-module and $N$ may be viewed as a right $\mathcal{A} \rtimes_{\alpha} G$-module.

Proof. Let $c \delta_{g}$ be in $M$, where $g \in G$ and $c \in \mathcal{A}$, and let $a \delta_{h} \in \mathcal{A} \rtimes_{\alpha} G$, where $h \in G$ and $a \in \mathcal{D}_{h}$. Then

$$
a \delta_{h} \cdot c \delta_{g}=a \beta_{h}(c) \delta_{h g} \in M,
$$

because $\mathcal{A}$ is an ideal in $M$. So $M$ is a left $\mathcal{A} \rtimes_{\alpha} G$-module.

Next let $c \delta_{g}$ be in $N$, where $g \in G$ and $c=\beta_{g}\left(c^{\prime}\right)$ with $c^{\prime} \in \mathcal{A}$, and let $a \delta_{h} \in$ $\mathcal{A} \rtimes_{\alpha} G$, where $h \in G$ and $a \in \mathcal{D}_{h}$. Then

$$
c \delta_{g} \cdot a \delta_{h}=c \beta_{g}(a) \delta_{g h}=\beta_{g}\left(c^{\prime} a\right) \delta_{g h}=\cdots .
$$

Since $\mathcal{D}_{h}$ is an ideal in $\mathcal{A}$, we have that $c^{\prime} a \in \mathcal{D}_{h}$ and hence $c^{\prime} a=\alpha_{h}(x)$ for some $x \in \mathcal{D}_{h^{-1}}$. So the above equals

$$
\cdots=\beta_{g}\left(\alpha_{h}(x)\right) \delta_{g h}=\beta_{g}\left(\beta_{h}(x)\right) \delta_{g h}=\beta_{g h}(x) \delta_{g h} \in N .
$$

So $N$ is a right $\mathcal{A} \rtimes_{\alpha} G$-module.

So far we have therefore seen that $M$ is an $\left(\mathcal{A} \rtimes_{\alpha} G\right)-\left(\mathcal{B} \rtimes_{\beta} G\right)$-bimodule, whereas $N$ is a $\left(\mathcal{B} \rtimes_{\beta} G\right)-\left(\mathcal{A} \rtimes_{\alpha} G\right)$-bimodule. These give the third and fourth components of the Morita context we are looking for. Next we need to find $\tau$ and $\tau^{\prime}$.

Given linear subspaces $X$ and $Y$ of an algebra, we will denote by $X Y$ the linear span of the set of products $x y$ with $x \in X$ and $y \in Y$. This notation will be used in our next result.

Proposition 5.3. $M N=\mathcal{A} \rtimes_{\alpha} G$ and $N M=\mathcal{B} \rtimes_{\beta} G$.

Proof. Let $c_{1} \delta_{g} \in M$ and $c_{2} \delta_{h} \in N$, where $g, h \in G, c_{1} \in \mathcal{A}$, and $c_{2}=\beta_{h}\left(c_{2}^{\prime}\right)$ with $c_{2}^{\prime} \in \mathcal{A}$. Then

$$
c_{1} \delta_{g} \cdot c_{2} \delta_{h}=c_{1} \beta_{g}\left(c_{2}\right) \delta_{g h}=c_{1} \beta_{g h}\left(c_{2}^{\prime}\right) \delta_{g h} \in \mathcal{A} \rtimes_{\alpha} G,
$$

because $c_{1} \beta_{g h}\left(c_{2}^{\prime}\right) \in \mathcal{A} \cap \beta_{g h}(\mathcal{A})=\mathcal{D}_{g h}$. This shows that $M N \subseteq \mathcal{A} \rtimes_{\alpha} G$.

Given $c \in \mathcal{D}_{h}$, observe that $c \in \alpha_{h}\left(\mathcal{D}_{h^{-1}}\right) \subseteq \beta_{h}(\mathcal{A})$, so that $c \delta_{h} \in N$. Letting $1_{\mathcal{A}}$ be the unit of $\mathcal{A}$, we have that $1_{\mathcal{A}} \delta_{e} \in M$ and

$$
1_{\mathcal{A}} \delta_{e} \cdot c \delta_{h}=1_{\mathcal{A}} c \delta_{h}=c \delta_{h} .
$$

So $c \delta_{h} \in M N$. Since $c$ is arbitrary, we conclude that $\mathcal{A} \rtimes_{\alpha} G \subseteq M N$.

Let $g, h \in G$ and let $c \in \mathcal{A}$. Then $\beta_{g}(c) \delta_{g} \in N$ and $1_{\mathcal{A}} \delta_{g^{-1} h} \in M$. Moreover,

$$
\beta_{g}(c) \delta_{g} \cdot 1_{\mathcal{A}} \delta_{g^{-1} h}=\beta_{g}\left(c 1_{\mathcal{A}}\right) \delta_{h}=\beta_{g}(c) \delta_{h} .
$$

Since $\bigcup_{g \in G} \beta_{g}(\mathcal{A})$ generates $\mathcal{B}$, we conclude that $\mathcal{B} \delta_{h} \subseteq N M$, and hence $N M=$ $\mathcal{B} \rtimes_{\beta} G$. 
The above result suggests that we define

$$
\tau: m \otimes n \in M \otimes_{\mathcal{B} \rtimes_{\beta} G} N \longmapsto m n \in \mathcal{A} \rtimes_{\alpha} G
$$

and

$$
\tau^{\prime}: n \otimes m \in N \otimes_{\mathcal{A} \rtimes_{\alpha} G} M \longmapsto n m \in \mathcal{B} \rtimes_{\beta} G .
$$

It is then easy to see that the six-tuple $\left(\mathcal{A} \rtimes_{\alpha} G, \mathcal{B} \rtimes_{\beta} G, M, N, \tau, \tau^{\prime}\right)$ is a Morita context. This leads us to the main result of this section:

Theorem 5.4. Let $\alpha$ be a partial action of a group $G$ on a unital algebra $\mathcal{A}$ and suppose that $(\beta, \mathcal{B})$ is an enveloping action for $(\alpha, \mathcal{A})$ such that $\mathcal{B}$ is also unital. Then $\mathcal{A} \rtimes_{\alpha} G$ and $\mathcal{B} \rtimes_{\beta} G$ are Morita equivalent.

Proof. By (5.3) we have that both $\tau$ and $\tau^{\prime}$ are onto. The conclusion then follows from [18, Theorem 4.1.17].

\section{Partial Representations and partial actions}

In this section we use crossed products to relate partial actions with partial representations of groups.

Definition 6.1. A partial representation of a group $G$ into a unital $K$-algebra $\mathcal{B}$ is a map

$$
\pi: G \rightarrow \mathcal{B}
$$

which sends the unit element of the group to the unity element of $\mathcal{B}$, and is such that for all $g, h \in G$ we have

$$
\pi(g) \pi(h) \pi\left(h^{-1}\right)=\pi(g h) \pi\left(h^{-1}\right) \quad \text { and } \quad \pi\left(g^{-1}\right) \pi(g) \pi(h)=\pi\left(g^{-1}\right) \pi(g h) .
$$

In particular, if $\mathcal{B}$ is the algebra of linear transformations $\operatorname{End}(V)$ of a vector space $V$ over a field $K$, then we have a partial representation of $G$ on the vector space $V$.

Lemma 6.2. Let $\alpha=\left\{\alpha_{g}: \mathcal{D}_{g^{-1}} \rightarrow \mathcal{D}_{g}(g \in G)\right\}$ be a partial action of $G$ on an algebra $\mathcal{A}$ such that each $\mathcal{D}_{g}(g \in G)$ is a unital algebra with unity $1_{g}$. Then the map $\pi_{\alpha}: G \ni g \mapsto 1_{g} \delta_{g} \in \mathcal{A} \rtimes_{\alpha} G$ is a partial representation.

Proof. Obviously, $1 \mapsto 1 \cdot \delta_{1}$, the identity element of $\mathcal{A} \rtimes_{\alpha} G$. We see that

$$
\begin{aligned}
& \left(1_{g^{-1}} \delta_{g^{-1}} \cdot 1_{g} \delta_{g}\right) \cdot 1_{h} \delta_{h}=\alpha_{g^{-1}}\left(\alpha_{g}\left(1_{g^{-1}}\right) 1_{g}\right) \delta_{1} \cdot 1_{h} \delta_{h}=\alpha_{g^{-1}}\left(1_{g}^{2}\right) \delta_{1} \cdot 1_{h} \delta_{h} \\
& =\alpha_{g^{-1}}\left(1_{g}\right) \delta_{1} \cdot 1_{h} \delta_{h}=1_{g^{-1}} \delta_{1} \cdot 1_{h} \delta_{h}=1_{g^{-1}} 1_{h} \delta_{h} .
\end{aligned}
$$

On the other hand, using (5), we have

$$
1_{g^{-1}} \delta_{g^{-1}} \cdot 1_{g h} \delta_{g h}=\alpha_{g^{-1}}\left(\alpha_{g}\left(1_{g^{-1}}\right) 1_{g h}\right) \delta_{h}=\alpha_{g^{-1}}\left(1_{g} 1_{g h}\right) \delta_{h}=1_{g^{-1}} 1_{h} \delta_{h}
$$

Thus $1_{g^{-1}} \delta_{g^{-1}} \cdot 1_{g} \delta_{g} \cdot 1_{h} \delta_{h}=1_{g^{-1}} \delta_{g^{-1}} \cdot 1_{g h} \delta_{g h}$ for all $g, h \in G$, and one similarly verifies that $1_{g} \delta_{g} \cdot 1_{h} \delta_{h} \cdot 1_{h^{-1}} \delta_{h^{-1}}=1_{g} 1_{g h} \delta_{g}=1_{g h} \delta_{g h} \cdot 1_{h^{-1}} \delta_{h^{-1}}(g, h \in G)$, which shows that $\pi_{\alpha}: G \rightarrow \mathcal{A} \rtimes_{\alpha} G$ is a partial representation.

Definition 6.3. Two partial representations $\pi: G \rightarrow \mathcal{B}$ and $\pi^{\prime}: G \rightarrow \mathcal{B}^{\prime}$ are equivalent if there is an isomorphism $\varphi: \mathcal{B}^{\prime} \rightarrow \mathcal{B}$ such that

$$
\pi(g)=\varphi\left(\pi^{\prime}(g)\right)
$$

for all $g \in G$.

Remark 6.4. It is easily verified that the map $\alpha \mapsto \pi_{\alpha}$ sends equivalent partial actions into equivalent partial representations. 
Let $\pi: G \rightarrow \mathcal{B}$ be a partial representation of a group $G$ into a unital $K$-algebra $\mathcal{B}$. Then by (2), (3) of [5] the elements $\varepsilon_{g}=\pi(g) \pi\left(g^{-1}\right)(g \in G)$ are commuting idempotents such that

$$
\pi(g) \varepsilon_{h}=\varepsilon_{g h} \pi(g), \quad \varepsilon_{h} \pi(g)=\pi(g) \varepsilon_{g^{-1} h} .
$$

Let $\mathcal{A}$ be the subalgebra of $\mathcal{B}$ generated by all the $\varepsilon_{g}(g \in G)$, and for a fixed $g \in G$ set $\mathcal{D}_{g}=\varepsilon_{g} \mathcal{A}$.

Lemma 6.5. The maps $\alpha_{g}^{\pi}: \mathcal{D}_{g^{-1}} \rightarrow \mathcal{D}_{g}(g \in G)$ defined by $\alpha_{g}^{\pi}(a)=\pi(g) a \pi\left(g^{-1}\right)$ $\left(a \in \mathcal{D}_{g^{-1}}\right)$ are isomorphisms of $K$-algebras which determine a partial action $\alpha^{\pi}$ of $G$ on $\mathcal{A}$.

Proof. Write for simplicity $\alpha^{\pi}=\alpha$. We observe first that $\mathcal{A}$ is invariant with respect to the map $a \mapsto \pi(g) a \pi\left(g^{-1}\right)$. Obviously, $\mathcal{A}$ is spanned by elements $a=\varepsilon_{h_{1}} \ldots \varepsilon_{h_{s}}$ with $h_{1}, \ldots, h_{s} \in G$. We see that

$$
\pi(g) \varepsilon_{h_{1}} \ldots \varepsilon_{h_{s}} \pi\left(g^{-1}\right)=\pi(g) \pi\left(g^{-1}\right) \varepsilon_{g h_{1}} \ldots \varepsilon_{g h_{s}}=\varepsilon_{g} \varepsilon_{g h_{1}} \ldots \varepsilon_{g h_{s}} \in \mathcal{A} .
$$

Thus $\pi(g) \mathcal{A} \pi\left(g^{-1}\right) \subseteq \mathcal{A}$. It is easily verified that this map sends $\varepsilon_{g^{-1}}$ to $\varepsilon_{g}$. Since $\mathcal{D}_{g}$ is generated in $\mathcal{A}$ by the idempotent $\varepsilon_{g}$, we obtain a $K$-map $\alpha_{g}: \mathcal{D}_{g^{-1}} \rightarrow \mathcal{D}_{g}$. Moreover, it is a homomorphism of algebras. Indeed, taking $a, b \in \mathcal{D}_{g^{-1}}$, we see that

$$
\begin{aligned}
\alpha_{g}(a) \alpha_{g}(b) & =\pi(g) a \pi\left(g^{-1}\right) \pi(g) b \pi\left(g^{-1}\right)=\pi(g) a \varepsilon_{g^{-1}} b \pi\left(g^{-1}\right) \\
& =\pi(g) a b \pi\left(g^{-1}\right)=\alpha_{g}(a b),
\end{aligned}
$$

as $\varepsilon_{g^{-1}}$ is the identity element of $\mathcal{D}_{g^{-1}}$. Then $\alpha_{g^{-1}}: \mathcal{D}_{g} \rightarrow \mathcal{D}_{g^{-1}}$ is also a homomorphism of algebras, and it is easily seen that $\alpha_{g} \circ \alpha_{g^{-1}}$ and $\alpha_{g^{-1}} \circ \alpha_{g}$ are identity maps.

Taking $g, h \in G$, write an element $a \in \mathcal{D}_{h} \cap \mathcal{D}_{g^{-1}}$ as $a=\varepsilon_{h} \varepsilon_{g^{-1}} b$ with $b \in \mathcal{A}$. Then

$$
\begin{aligned}
\alpha_{h}^{-1}(a) & =\pi\left(h^{-1}\right) \varepsilon_{h} \varepsilon_{g^{-1}} b \pi(h)=\pi\left(h^{-1}\right) \varepsilon_{h} \pi(h) \varepsilon_{h^{-1} g^{-1}} b^{\prime} \\
& =\varepsilon_{h^{-1}} \varepsilon_{h^{-1} g^{-1}} b^{\prime} \in \mathcal{D}_{(g h)^{-1}},
\end{aligned}
$$

with $b^{\prime} \in \mathcal{A}$. Hence $\mathcal{D}_{(g h)^{-1}} \supseteq \alpha_{h}^{-1}\left(\mathcal{D}_{h} \cap \mathcal{D}_{g^{-1}}\right)$, which is condition (ii) of Definition 1.1

Finally, for $a \in \alpha_{h}^{-1}\left(\mathcal{D}_{h} \cap \mathcal{D}_{g^{-1}}\right)$, we have $\varepsilon_{h^{-1}} a=a \varepsilon_{h^{-1}}=a$, as $a \in \mathcal{D}_{h^{-1}}$, and thus,

$$
\begin{aligned}
& \alpha_{g} \circ \alpha_{h}(a)=\pi(g) \pi(h) a \pi\left(h^{-1}\right) \pi\left(g^{-1}\right)=\pi(g) \pi(h) a \varepsilon_{h^{-1}} \pi\left(h^{-1}\right) \pi\left(g^{-1}\right) \\
& =\pi(g) \pi(h) a \varepsilon_{h^{-1}} \pi\left(h^{-1} g^{-1}\right)=\pi(g) \pi(h) \varepsilon_{h^{-1}} a \pi\left(h^{-1} g^{-1}\right) \\
& =\pi(g h) \varepsilon_{h^{-1}} a \pi\left(h^{-1} g^{-1}\right)=\alpha_{g h}(a),
\end{aligned}
$$

which gives (iii) of Definition 1.1. Since (i) is obvious, the lemma is proved.

Remark 6.6. As in the previous case, it is readily seen that $\pi \mapsto \alpha^{\pi}$ also preserves equivalence relations.

Proposition 6.7. Let $\alpha=\left\{\alpha_{g}: \mathcal{D}_{g^{-1}} \rightarrow \mathcal{D}_{g}(g \in G)\right\}$ be a partial action of $G$ on an algebra $\mathcal{A}$ such that each $\mathcal{D}_{g}(g \in G)$ is a unital algebra with unity $1_{g}$. Let $\mathcal{A}^{\prime}$ be the subalgebra of $\mathcal{A} \rtimes_{\alpha} G$ generated by all $1_{g} \delta_{1}(g \in G)$. Then the map $\varphi_{\alpha}: \mathcal{A}^{\prime} \ni 1_{g} \delta_{1} \mapsto 1_{g} \in \mathcal{A}$ is a monomorphism such that $\varphi_{\alpha} \circ \alpha_{g}^{\pi_{\alpha}}=\alpha_{g} \circ \varphi_{\alpha}$ for each $g \in G$. In particular, if $\mathcal{A}$ is generated by the elements $1_{g}(g \in G)$, then the partial actions $\alpha^{\pi_{\alpha}}$ and $\alpha$ are equivalent. 
Proof. Clearly, $\varphi_{\alpha}$ is the restriction of the isomorphism $\mathcal{A} \cdot \delta_{1} \ni a \delta_{1} \mapsto a \in \mathcal{A}$ and thus is a monomorphism of $\mathcal{A}^{\prime}$ into $\mathcal{A}$.

According to Lemma 6.2 we have the partial representation $\pi_{\alpha}: G \ni g \mapsto 1_{g} \delta_{g} \in$ $\mathcal{A} \rtimes_{\alpha} G$, which by Lemma 6.5 induces a partial action on the subalgebra of $\mathcal{A} \rtimes_{\alpha} G$ generated by the elements $\pi_{\alpha}(g) \pi_{\alpha}\left(g^{-1}\right)$. Because

$$
\pi_{\alpha}(g) \pi_{\alpha}\left(g^{-1}\right)=1_{g} \delta_{g} 1_{g^{-1}} \delta_{g^{-1}}=\alpha_{g}\left(\alpha_{g^{-1}}\left(1_{g}\right) 1_{g^{-1}}\right) \delta_{1}=1_{g} \delta_{1},
$$

this subalgebra is exactly $\mathcal{A}^{\prime}$. An arbitrary element of $\mathcal{A}^{\prime}$ can be written as $a^{\prime} \delta_{1}$, where $a^{\prime}$ belongs to the image $\operatorname{Im} \varphi_{\alpha}$ of $\varphi_{\alpha}$. The partial action $\alpha^{\pi_{\alpha}}$ is given by the isomorphisms $\alpha^{\pi_{\alpha}}: \mathcal{D}_{g^{-1}}^{\prime} \ni a^{\prime} \delta_{1} \mapsto 1_{g} \delta_{g} \cdot a^{\prime} \cdot 1_{g^{-1}} \delta_{g^{-1}} \in \mathcal{D}_{g}^{\prime}$, where $\mathcal{D}_{g}^{\prime}$ is the ideal of $\mathcal{A}^{\prime}$ generated by $1_{g} \delta_{1}$. For $a^{\prime} \delta_{1} \in \mathcal{D}_{g^{-1}}^{\prime}$ we see that

$$
\begin{aligned}
& \varphi_{\alpha}\left(\alpha_{g}^{\pi_{\alpha}}\left(a^{\prime} \delta_{1}\right)\right)=\varphi_{\alpha}\left(1_{g} \delta_{g} a^{\prime} 1_{g^{-1}} \delta_{g^{-1}}\right)=\varphi_{\alpha}\left(\alpha_{g}\left(\alpha_{g^{-1}}\left(1_{g}\right) a^{\prime} 1_{g^{-1}}\right) \delta_{1}\right) \\
& =\varphi_{\alpha}\left(\alpha_{g}\left(a^{\prime} 1_{g^{-1}}\right) \delta_{1}\right)=\alpha_{g}\left(a^{\prime}\right)=\alpha_{g}\left(\varphi_{\alpha}\left(a^{\prime} \delta_{1}\right)\right),
\end{aligned}
$$

so that $\varphi_{\alpha} \circ \alpha_{g}^{\pi_{\alpha}}=\alpha_{g} \circ \varphi_{\alpha}$ for all $g \in G$. If $\mathcal{A}$ is generated by the elements $1_{g}$ $(g \in G)$, then clearly $\varphi_{\alpha}: \mathcal{A}^{\prime} \rightarrow \mathcal{A}$ is an isomorphism which gives the equivalence of the partial actions $\alpha^{\pi_{\alpha}}$ and $\alpha$.

Proposition 6.8. Let $\pi: G \rightarrow \mathcal{B}$ be a partial representation and suppose that the subalgebra $\mathcal{A} \subseteq \mathcal{B}$ and the partial action $\alpha^{\pi}$ of $G$ on $\mathcal{A}$ are as in Lemma 6.5. Then the map $\phi_{\pi}: \mathcal{A} \rtimes_{\alpha^{\pi}} G \rightarrow \mathcal{B}$ defined by $\phi_{\pi}\left(\sum_{g \in G} a_{g} \delta_{g}\right)=\sum_{g \in G} a_{g} \pi(g)$ is a homomorphism of $K$-algebras such that $\phi_{\pi} \circ \pi_{\alpha^{\pi}}=\pi$. In particular, if $\phi_{\pi}$ is an isomorphism, then the partial representations $\pi$ and $\pi_{\alpha^{\pi}}$ are equivalent.

Proof. Obviously, the elements $a_{g} \delta_{g}\left(g \in G, a_{g} \in \mathcal{D}_{g}\right)$ span $\mathcal{A} \rtimes_{\alpha^{\pi}} G$. Since $\varepsilon_{g}$ is the identity element of $\mathcal{D}_{g}$ and $\varepsilon_{g} \pi(g h)=\varepsilon_{g} \pi(g) \pi(h)$, we have

$$
\begin{aligned}
& \phi_{\pi}\left(a_{g} \delta_{g} \cdot b_{h} \delta_{h}\right) \\
& =\phi_{\pi}\left(\alpha_{g}^{\pi}\left(\alpha_{g^{-1}}^{\pi}\left(a_{g}\right) b_{h}\right) \delta_{g h}\right)=\alpha_{g}^{\pi}\left(\alpha_{g^{-1}}^{\pi}\left(a_{g}\right) b_{h}\right) \pi(g h)=\alpha_{g}^{\pi}\left(\alpha_{g^{-1}}^{\pi}\left(a_{g}\right) b_{h}\right) \varepsilon_{g} \pi(g h) \\
& =\alpha_{g}^{\pi}\left(\alpha_{g^{-1}}^{\pi}\left(a_{g}\right) b_{h}\right) \varepsilon_{g} \pi(g) \pi(h)=\alpha_{g}^{\pi}\left(\alpha_{g^{-1}}^{\pi}\left(a_{g}\right) b_{h}\right) \pi(g) \pi(h) \\
& =\pi(g)\left(\alpha_{g^{-1}}^{\pi}\left(a_{g}\right) b_{h}\right) \pi\left(g^{-1}\right) \pi(g) \pi(h)=\pi(g) \alpha_{g^{-1}}^{\pi}\left(a_{g}\right) b_{h} \varepsilon\left(g^{-1}\right) \pi(h) \\
& =\pi(g) \alpha_{g^{-1}}^{\pi}\left(a_{g}\right) b_{h} \pi(h),
\end{aligned}
$$

as $\alpha_{g^{-1}}^{\pi}\left(a_{g}\right) b_{h} \in \mathcal{D}_{g^{-1}}$. Continuing this calculation, we see that

$$
\begin{aligned}
& \pi(g) \alpha_{g^{-1}}^{\pi}\left(a_{g}\right) b_{h} \pi(h)=\pi(g) \pi\left(g^{-1}\right) a_{g} \pi(g) b_{h} \pi(h)=\varepsilon(g) a_{g} \pi(g) b_{h} \pi(h) \\
& =a_{g} \pi(g) b_{h} \pi(h)=\phi_{\pi}\left(a_{g} \delta_{g}\right) \cdot \phi_{\pi}\left(b_{h} \delta_{h}\right),
\end{aligned}
$$

using once more that $\varepsilon_{g}$ is the identity of $\mathcal{D}_{g}$. Thus, $\phi_{\pi}: \mathcal{A} \rtimes_{\alpha^{\pi}} G \rightarrow \mathcal{B}$ is a homomorphism of algebras. It is easily seen that $\phi_{\pi} \circ \pi_{\alpha^{\pi}}(g)=\phi_{\pi}\left(\varepsilon_{g} \delta_{g}\right)=\varepsilon_{g} \pi(g)=$ $\pi(g) \pi\left(g^{-1}\right) \pi(g)=\pi(g)$, for each $g \in G$, which shows that $\phi_{\pi} \circ \pi_{\alpha^{\pi}}=\pi$. If $\phi_{\pi}$ is an isomorphism, then it obviously follows that $\pi$ and $\pi_{\alpha^{\pi}}$ are equivalent.

Now we can show that the partial group algebras can be naturally endowed with a crossed product structure. We remind the reader that the partial group algebra $K_{\text {par }}(G)$ of a group $G$ over $K$ is the semigroup algebra $K S(G)$, where $S(G)$ is the universal semigroup, generated by the symbols $\{[g]: g \in G\}$ subject to the relations

a) $\left[g^{-1}\right][g][h]=\left[g^{-1}\right][g h]$;

b) $[g][h]\left[h^{-1}\right]=[g h]\left[h^{-1}\right] \quad(g, h \in G)$;

c) $[1]=1$, 
where 1 also denotes the identity element of $S(G)$. It is easily seen that $\tilde{\pi}: G \ni g \mapsto$ $[g] \in K_{\text {par }}(G)$ is a partial representation and $K_{\text {par }}(G)$ has the following universal property: for every partial representation $\pi$ of $G$ into a unital algebra $\mathcal{B}$ there exists a unique homomorphism of algebras $\psi: K_{\text {par }}(G) \rightarrow \mathcal{B}$ such that $\pi=\psi \circ \tilde{\pi}$, and, conversely, for each algebra homomorphism $\psi: K_{\text {par }}(G) \rightarrow \mathcal{B}$ the map $\psi \circ \tilde{\pi}$ is a partial representation of $G$ into $\mathcal{B}$.

Theorem 6.9. Let $\mathcal{A}$ be the subalgebra of $K_{\mathrm{par}}(G)$ generated by the elements $\tilde{\varepsilon}_{g}=\tilde{\pi}(g) \tilde{\pi}\left(g^{-1}\right)=[g]\left[g^{-1}\right]$. Then the homomorphism $\phi_{\tilde{\pi}}: \mathcal{A} \rtimes_{\alpha \tilde{\pi}} G \rightarrow K_{\mathrm{par}}(G)$ associated to the partial representation $\tilde{\pi}: G \ni g \mapsto[g] \in K_{\mathrm{par}}(G)$ is actually an isomorphism.

Proof. By Proposition 6.8, $\phi_{\tilde{\pi}}: \mathcal{A} \rtimes_{\alpha^{\tilde{\pi}}} G \rightarrow K_{\mathrm{par}}(G)$ is a homomorphism. We shall show that it has an inverse. Recall that $\mathcal{D}_{g}$ is spanned by the elements of the form $\tilde{\varepsilon}_{g} \cdot \tilde{\varepsilon}_{h_{1}} \cdot \ldots \cdot \tilde{\varepsilon}_{h_{s}}$. By the universal property of partial group rings, the partial representation $\pi_{\alpha^{\tilde{\pi}}}: G \ni g \mapsto \tilde{\varepsilon}_{g} \delta_{g} \in \mathcal{A} \rtimes_{\alpha^{\tilde{\pi}}} G$ gives a homomorphism $\psi: K_{\mathrm{par}}(G) \rightarrow \mathcal{A} \rtimes_{\alpha \tilde{\pi}} G$ such that $\psi([g])=\tilde{\varepsilon}_{g} \delta_{g}$.

Obviously, $\phi_{\tilde{\pi}} \circ \psi[g]=\phi_{\tilde{\pi}}\left(\tilde{\varepsilon}_{g} \delta_{g}\right)=\tilde{\varepsilon}_{g}[g]=[g]\left[g^{-1}\right][g]=[g]$ for each $g \in G$, so that $\phi_{\tilde{\pi}} \circ \psi$ is the identity map. On the other hand, since $\tilde{\varepsilon}_{g} \delta_{g} \tilde{\varepsilon}_{g^{-1}} \delta_{g^{-1}}=\tilde{\varepsilon}_{g} \delta_{1}$, we have

$$
\begin{aligned}
& \psi \circ \phi_{\tilde{\pi}}\left(\left(\tilde{\varepsilon}_{g} \cdot \tilde{\varepsilon}_{h_{1}} \cdot \ldots \cdot \tilde{\varepsilon}_{h_{s}}\right) \delta_{g}\right)=\psi\left(\left(\tilde{\varepsilon}_{g} \cdot \tilde{\varepsilon}_{h_{1}} \cdot \ldots \cdot \tilde{\varepsilon}_{h_{s}}\right)[g]\right) \\
& =\psi\left([g]\left[g^{-1}\right]\left[h_{1}\right]\left[h_{1}^{-1}\right] \ldots\left[h_{s}\right]\left[h_{s}^{-1}\right][g]\right) \\
& =\left(\tilde{\varepsilon}_{g} \delta_{g} \tilde{\varepsilon}_{g^{-1}} \delta_{g^{-1}}\right)\left(\tilde{\varepsilon}_{h_{1}} \delta_{h_{1}} \tilde{\varepsilon}_{h_{1}^{-1}} \delta_{h_{1}^{-1}}\right) \ldots\left(\tilde{\varepsilon}_{h_{s}} \delta_{h_{s}} \tilde{\varepsilon}_{h_{s}^{-1}} \delta_{h_{s}^{-1}}\right) \tilde{\varepsilon}_{g} \delta_{g} \\
& =\tilde{\varepsilon}_{g} \delta_{1} \tilde{\varepsilon}_{h_{1}} \delta_{1} \ldots \tilde{\varepsilon}_{h_{s}} \delta_{1} \tilde{\varepsilon}_{g} \delta_{g}=\tilde{\varepsilon}_{g}^{2} \tilde{\varepsilon}_{h_{1}} \ldots \tilde{\varepsilon}_{h_{s}} \delta_{g}=\tilde{\varepsilon}_{g} \tilde{\varepsilon}_{h_{1}} \ldots \tilde{\varepsilon}_{h_{s}} \delta_{g} .
\end{aligned}
$$

Thus $\psi \circ \phi_{\tilde{\pi}}$ is also the identity map, and consequently $\psi$ is the inverse of $\phi_{\tilde{\pi}}$.

The next fact helps to identify algebras as crossed products.

Proposition 6.10. Let $\alpha=\left\{\alpha_{g}: \mathcal{D}_{g^{-1}} \rightarrow \mathcal{D}_{g}(g \in G)\right\}$ be a partial action of $G$ on an algebra $\mathcal{A}$ such that for each $g \in G$ the right annihilator of $\mathcal{D}_{g}$ in $\mathcal{D}_{g}$ is zero. Suppose that $\varphi: \mathcal{A} \rtimes_{\alpha} G \rightarrow \mathcal{B}$ is a homomorphism of algebras whose restriction to $\mathcal{A}$ is injective. If there exists a K-linear transformation $E: \mathcal{B} \rightarrow \mathcal{B}$ such that for every $g \in G, a \in \mathcal{D}_{g}$

$$
E\left(\varphi\left(a \delta_{g}\right)\right)= \begin{cases}\varphi\left(a \delta_{g}\right) & \text { if } g=1 \\ 0 & \text { if } g \neq 1\end{cases}
$$

then $\varphi$ is injective.

Proof. Suppose that $\sum_{g \in G} a_{g} \delta_{g}$ belongs to the kernel $\operatorname{Ker} \varphi$ of $\varphi$. Then for each $b \in \mathcal{D}_{h^{-1}}$ we have $\sum a_{g} \delta_{g} \cdot b \delta_{h^{-1}} \in \operatorname{Ker} \varphi$ and

$$
0=\varphi\left(\sum_{g \in G} a_{g} \delta_{g} \cdot b \delta_{h^{-1}}\right)=\varphi\left(\sum_{g \in G} \alpha_{g}\left(\alpha_{g^{-1}}\left(a_{g}\right) b\right) \delta_{g h^{-1}}\right) .
$$

Applying $E$, we eliminate all terms with $g \neq h$, so that

$$
\varphi\left(\alpha_{h}\left(\alpha_{h^{-1}}\left(a_{h}\right) b\right) \delta_{1}\right)=0 .
$$

Because $\varphi$ is injective on $\mathcal{A}\left(=\mathcal{A} \delta_{1}\right)$, we obtain

$$
0=\alpha_{h}\left(\alpha_{h^{-1}}\left(a_{h}\right) b\right)=a_{h} \alpha_{h}(b)
$$


for every $b \in \mathcal{D}_{h^{-1}}$. Since $\alpha_{h}(b)$ runs over $\mathcal{D}_{h}$ and the annihilator of $\mathcal{D}_{h}$ in $\mathcal{D}_{h}$ is zero, we conclude that $a_{h}=0$ for each $h \in G$. This yields that $\varphi$ is a monomorphism.

We apply the above results to matrix partial representations. First we recall some facts obtained in [5] and [6] (see also 7]). Let $A$ be a subset of a group $G$ which contains 1 and let $H$ be the stabilizer of $A$ in $G$ with respect to multiplication from the left, that is, $H=S t(A)=\{h \in G: h A=A\}$. Then $A$ is a union of right cosets of $H$. Suppose that $A$ is a finite union of such cosets, i.e., the index $(A: H)$ of $H$ in $A$ is finite, say $n$. Then left multiplication of $A$ by the elements $g$ with $g^{-1} \in A$ gives a finite number of distinct sets $A=A_{1}, A_{2}, \ldots, A_{n}$, each of which contains 1 . Consider the category $\Gamma$ whose objects are the sets $A_{1}, \ldots, A_{n}$ and whose morphisms are as follows. If $A_{i}$ and $A_{j}$ are given and there is some $g \in G$ such that $g A_{i}=A_{j}$ (in which case $g^{-1}$ necessarily belongs to $A_{i}$ ), then $\operatorname{Hom}\left(A_{i}, A_{j}\right)$ is the set of all pairs $\left(A_{i}, g\right)$ with $g$ as above. Otherwise $\operatorname{Hom}\left(A_{i}, A_{j}\right)$ is the empty set. A morphism can be viewed as left multiplication of $A_{i}$ by $g$, where $g^{-1} \in A_{i}$. The product $\left(A_{i}, g\right) \circ\left(A_{j}, g^{\prime}\right)$ is defined in $\Gamma$ if and only if $g^{\prime} A_{j}=A_{i}$, and if so, it is equal to $\left(A_{j}, g g^{\prime}\right)$. The identity morphism of $A_{i}$ is clearly $\left(A_{i}, 1\right)$, and, because each morphism has an inverse, $\Gamma$ is a groupoid. The subgroup $H$ is the isotropy group of $A$, and the isotropy group of $A_{i}$ is conjugate to $H$ in $G$. Identify $\Gamma$ with the set of its morphisms. Then the groupoid algebra $K \Gamma$ is the $K$-vector space with basis $\Gamma$ endowed with the following multiplication:

$$
\gamma_{1} \cdot \gamma_{2}= \begin{cases}\gamma_{1} \gamma_{2}, & \text { if the composite morphism } \gamma_{1} \gamma_{2} \text { exists in } \Gamma \\ 0, & \text { otherwise. }\end{cases}
$$

By [5. Proposition 3.1], the groupoid algebra $K \Gamma$ is isomorphic to the algebra $M_{n}(K H)$ of $n \times n$ matrices over the group algebra $K H$, and an isomorphism $\tau$ : $K \Gamma \rightarrow M_{n}(K H)$ can be obtained as follows. For each $A_{i}$, fix an element $g_{i} \in G$ such that $g_{i} A=A_{i}$. If $g A_{i}=A_{j}$, we see that $g_{j}^{-1} g g_{i}=h$ for some $h \in H$. Then the map $\tau$ is given by $\left(A_{i}, g\right) \mapsto e_{j, i}(h)$, where $e_{j, i}(h)$ is the elementary matrix whose unique non-zero entry is $h \in H$, which is placed in the intersection of the $j$-th row and $i$-th column. Consider also the map $\lambda: G \rightarrow K \Gamma$, defined by

$$
\lambda(g)= \begin{cases}\sum_{A_{i} \ni g^{-1}}\left(A_{i}, g\right), & \text { if } g^{-1} \in \bigcup_{i} A_{i}, \\ 0, & \text { otherwise. }\end{cases}
$$

According to [7], $\lambda$ is a partial representation of $G$ into $K \Gamma$. If $\sigma^{\prime}: M_{n}(K H) \rightarrow$ $M_{n m}(K)$ is the representation extended from an irreducible representation $\sigma: H \rightarrow$ $M_{m}(K)$, then $\sigma^{\prime} \circ \tau \circ \lambda: G \rightarrow M_{n m}(K)$ is an irreducible partial representation and, moreover, every irreducible partial matrix $K$-representation of $G$ is equivalent to a partial representation of the form $\sigma^{\prime} \circ \tau \circ \lambda$ for some subset $A \ni 1$ of $G$ and some irreducible matrix $K$-representation $\sigma$ of the isotropy group $H$ of $A$. Thus the "partial part" of an irreducible finite dimensional partial $K$-representation is given by the partial representation $\tau \circ \lambda: G \rightarrow M_{n}(K H)$. The partial representations of the form $\tau \circ \lambda$ obtained from the subsets $A \ni 1$ shall be called the elementary partial representations of $G$. Different choices of the elements $g_{i}$ with $A_{i}=g_{i} A$ give rise to equivalent partial representations. By the equivalence class of an elementary partial representation we mean the set of all partial representations of $G$ which are equivalent to the given elementary partial representation. An elementary partial representation has trivial isotropy if the corresponding isotropy group $H$ is 1 . 
We shall see that an elementary partial representation $\pi: G \rightarrow M_{n}(K H)$ gives rise to a certain partial action of $G$ on the diagonal subalgebra $\operatorname{diag}(K, \ldots, K)$ of the full matrix algebra $M_{n}(K)$. This diagonal algebra is obviously isomorphic to $K^{n}$, the $n$-th direct power of $K$, so we shall speak about partial actions on $K^{n}$. Given a partial action $\alpha$ of $G$ on $\operatorname{diag}(K, \ldots, K) \cong K^{n}$, we see that each $\mathcal{D}_{g}$ is an algebra with unity $1_{g}$ which is a sum of some minimal idempotents $e_{i, i}(1)$. Set $A_{i}(\alpha)=\left\{g \in G:\left(1_{g}\right)_{i, i} \neq 0\right\}$, where $\left(1_{g}\right)_{i, i}$ denotes the $i$-th diagonal entry of $1_{g}$.

Theorem 6.11. For a fixed $n>0$ and a fixed subgroup $H$ of a group $G$ the maps $\pi \mapsto \alpha^{\pi}$ and $\alpha \mapsto \pi_{\alpha}$ establish a one-to-one correspondence between the equivalence classes of the elementary partial representations $\pi: G \rightarrow M_{n}(K H)$ and the equivalence classes of the partial actions $\alpha$ of $G$ on $K^{n}$ with $\operatorname{St}\left(A_{1}(\alpha)\right)=H$ and $\left(A_{1}(\alpha): H\right)=n$.

Proof. Let $\pi: G \rightarrow M_{n}(K H)$ be an elementary partial representation, and $\mathcal{A}$ the subalgebra generated by all $\varepsilon_{g}(g \in G)$. Then there is a subset $A \ni 1$ in $G$ with isotropy group $H$ such that $H$ has index $n$ in $A$ and $\pi$ is of form $\tau \circ \lambda$ with $\lambda$ and $\tau$ described above. Thus $\tau$ is determined by a fixed choice of the elements $g_{i} \in G$ with $A_{i}=g_{i} A$. It is easily seen that each matrix $\pi(g)$ is "monomial over $H$ ", i.e., each row and each column of $\pi(g)$ contains at most one non-zero entry, which is an element of $H$ (observe that zero rows and zero columns are allowed). It follows that $\varepsilon_{g}=\sum_{A_{i} \ni g} e_{i i}(1)$. For every $i, k \in\{1, \ldots, n\}$ with $k \neq i$ there exists $g^{\prime} \in A_{i}$ such that $g^{\prime} \notin A_{k}$, and we see that the $k$-th diagonal entry of the matrix $\varepsilon_{g} \varepsilon_{g^{\prime}}$ is zero. Hence for a fixed $i$ we have $\prod_{g \in A_{i}} \varepsilon_{g}=e_{i i}(1)$, and consequently $\mathcal{A}$ coincides with the diagonal subalgebra $\operatorname{diag}(K, \ldots, K)$ of $M_{n}(K)$. Thus by Lemma 6.5] $\alpha^{\pi}$ is a partial action of $G$ on $\mathcal{A} \cong K^{n}$, and we obviously see that $A_{1}\left(\alpha^{\pi}\right)=A_{1}=A$, so that $S t\left(A_{1}\left(\alpha^{\pi}\right)\right)=H$ (note that $\left.\varepsilon_{g}=1_{g}\right)$.

Next we want to show that $\phi_{\pi}: \mathcal{A} \rtimes_{\alpha^{\pi}} G \rightarrow M_{n}(K H)$, defined in Proposition 6.8 is an isomorphism. Observe first that $\phi_{\pi}$ is an epimorphism. Indeed, we saw already that the diagonal subalgebra $\operatorname{diag}(K, \ldots, K)$ is contained in the image $\operatorname{Im}\left(\phi_{\pi}\right)$ of $\phi_{\pi}$. Moreover, for $g \in G$ we have $\mathcal{D}_{g}=\bigoplus_{A_{i} \ni g} K e_{i, i}(1)$. Fix arbitrarily $h \in H$, $i, j \in\{1, \ldots, n\}$, and take $g=g_{i} h g_{j}{ }^{-1}$. Then $g \in A_{i}$, and thus $e_{i, i}(1) \in \mathcal{D}_{g}$, so that $e_{i, i}(1) \delta_{g}$ is an element of $\mathcal{A} \rtimes_{\alpha^{\pi}} G$. We see that the unique non-zero entry in the $i$-th row of $\pi(g)=\pi\left(g_{i} h g_{j}{ }^{-1}\right)$ is $h$, and it is placed in the intersection with the $j$-th column. Hence $\phi_{\pi}\left(e_{i, i}(1) \delta_{g}\right)=e_{i, i}(1) \pi(g)=e_{i, j}(h) \in \operatorname{Im}\left(\phi_{\pi}\right)$, and because this holds for all $h \in H$ and all $i, j \in\{1, \ldots, n\}$, it follows that $\operatorname{Im}\left(\phi_{\pi}\right)=M_{n}(K H)$.

For an element $x=\sum_{h \in H} x(h) h \in K H$ set $\operatorname{tr} x=x(1)$ and consider the linear transformation $E: M_{n}(K H) \ni\left(x_{i, j}\right) \mapsto\left(\operatorname{tr} x_{1,1}, \ldots, \operatorname{tr} x_{n, n}\right) \in \operatorname{diag}(K, \ldots, K) \subseteq$ $M_{n}(K)$. It is easily seen that if $g \neq 1$, then the only possible diagonal entries of $a \pi(g)\left(a \in \mathcal{D}_{g}\right)$ are non-identity elements of $H$ multiplied by scalars from $K$. Thus $E\left(\phi_{\pi}\left(a \delta_{g}\right)\right)=0$ for all $1 \neq g \in G$ and $a \in \mathcal{D}_{g}$, and moreover $E\left(\phi_{\pi}\left(a \delta_{1}\right)\right)=$ $\phi_{\pi}\left(a \delta_{1}\right)$, for each $a \in \mathcal{A}$. Since the restriction of $\phi_{\pi}$ to $\mathcal{A}$ is injective, it follows from Proposition 6.10 that $\phi_{\pi}$ is a monomorphism. Hence $\phi_{\pi}$ is an isomorphism, and by Proposition 6.8 the partial representations $\pi$ and $\pi_{\alpha^{\pi}}$ are equivalent.

Suppose now that $\alpha=\left\{\alpha_{g}: \mathcal{D}_{g^{-1}} \rightarrow \mathcal{D}_{g}(g \in G)\right\}$ is a partial action of $G$ on $K^{n}$ such that $S t\left(A_{1}(\alpha)\right)=H$ and the index of $H$ in $A_{1}(\alpha)$ is $n$. We are going to construct an elementary partial representation whose $\varepsilon_{g}$ 's will be exactly the $1_{g}$ 's. Suppose for a moment that $G$ is finite and let $S$ be a subset of $G$. One of the important working tools in $[5]$ is the product $\prod_{g \in S} \varepsilon_{g} \prod_{g \notin S}\left(1-\varepsilon_{g}\right)$. Replacing $S$ 
and $G \backslash S$ by certain finite subsets, it is possible to use this kind of product also for infinite $G$ 's, as it is done in the proof of Theorem 1 of [7]. We proceed by adopting these ideas to our situation. Let $G$ be again arbitrary and $S \ni 1$ be a subset of $G$. Each $1_{g}$ is an idempotent in $K^{n}$, and because this algebra has only a finite number of idempotents, we can choose finite subsets $S^{\prime} \subset S$ and $S^{\prime \prime} \subset G \backslash S$ such that $\left\{1_{g}: g \in S\right\}=\left\{1_{g}: g \in S^{\prime}\right\}$ and $\left\{1_{g}: g \in G \backslash S\right\}=\left\{1_{g}: g \in S^{\prime \prime}\right\}$. Set

$$
f_{S}=\prod_{g \in S^{\prime}} 1_{g} \prod_{g \in S^{\prime \prime}}\left(1-1_{g}\right) .
$$

Write for simplicity $A_{i}=A_{i}(\alpha)$. It follows from the definition of the $A_{i}(\alpha)$ 's that

$$
1_{g}=\sum_{A_{i} \ni g} e_{i, i}(1)
$$

We see that $f_{A_{i}} \neq 0$ for each $i=1, \ldots, n$, as $\left(f_{A_{i}}\right)_{i i}=e_{i, i}(1)$. Moreover,

$$
f_{S} \neq 0 \text { if and only if } S=A_{i} \text { for some } 1 \leq i \leq n \text {. }
$$

Indeed, suppose that $S \neq A_{j}$ for all $j=1, \ldots, n$ and fix $i \in\{1, \ldots, n\}$ arbitrarily. Then either there exists $g \in S$ with $g \notin A_{i}$ or there is an element $t \in A_{i}$ such that $t \notin S$. In the first case $\left(1_{g}\right)_{i i}=0$ and hence $\left(f_{S}\right)_{i i}=0$. In the second, $\left(f_{S}\right)_{i i}=0$ because $\left(1_{t}\right)_{i i}=1$ implies $\left(1-1_{t}\right)_{i i}=0$, where 1 is the identity element of $K^{n}=\operatorname{diag}(K, \ldots, K)$. Since $i$ is arbitrary, it follows that $f_{S}=0$.

Next we compute the effect of $\alpha_{g}$ on $f_{S}$, where $g^{-1} \in S$. It follows from the definition of $f_{S}$ that taking larger $S^{\prime}$ and $S^{\prime \prime}$ does not alter $f_{S}$. Thus we can choose $S^{\prime}$ and $S^{\prime \prime}$ in the definition of $f_{S}$ such that the conditions $\left\{1_{h}: h \in g S^{\prime}\right\}=\left\{1_{h}\right.$ : $h \in g S\}$ and $\left\{1_{t}: t \in g S^{\prime \prime}\right\}=\left\{1_{t}: t \in G \backslash g S\right\}$ are also guaranteed. Moreover, since $1 \in S$ we may clearly suppose that $S^{\prime} \ni 1$. By (5) $\alpha_{g}\left(1_{g^{-1}} 1_{h}\right)=1_{g} 1_{g h}$ for $g, h \in G$. Hence for $g, h_{1}, \ldots, h_{s} \in G$ we see that

$$
\begin{aligned}
& \alpha_{g}\left(1_{g^{-1}} 1_{h_{1}} 1_{h_{2}} \ldots 1_{h_{s}}\right)=\alpha_{g}\left(\left(1_{g^{-1}} 1_{h_{1}}\right)\left(1_{g^{-1}} 1_{h_{2}}\right) \ldots\left(1_{g^{-1}} 1_{h_{s}}\right)\right) \\
& =\alpha_{g}\left(1_{g^{-1}} 1_{h_{1}}\right) \alpha_{g}\left(1_{g^{-1}} 1_{h_{2}}\right) \ldots \alpha_{g}\left(1_{g^{-1}} 1_{h_{s}}\right)=\left(1_{g} 1_{g h_{1}}\right)\left(1_{g} 1_{g h_{2}}\right) \ldots\left(1_{g} 1_{g h_{s}}\right) \\
& =1_{g} 1_{g h_{1}} 1_{g h_{2}} \ldots 1_{g h_{s}} .
\end{aligned}
$$

Consequently, for $g \in G$ with $g^{-1} \in S$ we obtain

$$
\begin{aligned}
& \alpha_{g}\left(f_{S}\right)=\alpha_{g}\left(1_{g^{-1}} \prod_{h \in S^{\prime}} 1_{h} \prod_{t \in S^{\prime \prime}}\left(1-1_{t}\right)\right)=1_{g} \prod_{h \in S^{\prime}} 1_{g h} \prod_{t \in S^{\prime \prime}}\left(1-1_{g t}\right) \\
& =\prod_{h \in g S^{\prime}} 1_{h} \prod_{t \in g S^{\prime \prime}}\left(1-1_{t}\right)=f_{g S} .
\end{aligned}
$$

Thus we have

$$
\alpha_{g}\left(f_{S}\right)=f_{g S} \quad\left(g^{-1} \in S\right) .
$$

According to our assumption, $S t\left(A_{1}\right)=H$ has index $n$ in $A_{1}$. Therefore there exist elements $g_{1}, \ldots, g_{n} \in G$ with $g_{1}{ }^{-1}, \ldots g_{n}{ }^{-1} \in A_{1}$ such that we have $n$ distinct sets $g_{1} A_{1}, \ldots, g_{n} A_{1}$. It follows from (11) and (12) that up to a permutation these are exactly the sets $A_{1}, A_{2}, \ldots, A_{n}$. Hence we may suppose that $g_{i} A_{1}=A_{i}(i=$ $1, \ldots, n)$. In particular, $A_{i} \neq A_{j}$ if $1 \leq i \neq j \leq n$. Similarly as in the proof of (11) we easily see that $\left(f_{A_{i}}\right)_{j j}=0$ for every $j \neq i$, and consequently $f_{A_{i}}=e_{i, i}(1)$ for each $i=1, \ldots, n$.

Let $\pi^{\prime}$ be the elementary partial representation of $G$ determined by the set $A_{1}$. Then by (10), $\varepsilon_{g}^{\prime}=\pi^{\prime}(g) \pi^{\prime}\left(g^{-1}\right)=\sum_{A_{i} \ni g} e_{i, i}(1)=1_{g}$ for every $g \in G$, and 
the isomorphism $\alpha_{g}^{\pi^{\prime}}$ has the same domain as $\alpha_{g}$. Moreover, the algebra $K^{n} \equiv$ $\operatorname{diag}(K, \ldots, K)$ is generated by the $1_{g}$ 's, and for arbitrary $g, h_{1}, \ldots, h_{s} \in G$ we have

$$
\begin{aligned}
& \alpha_{g}^{\pi^{\prime}}\left(1_{g^{-1}} 1_{h_{1}} \ldots 1_{h_{s}}\right)=\pi^{\prime}(g) \varepsilon_{g^{-1}}^{\prime} \varepsilon_{h_{1}}^{\prime} \ldots \varepsilon_{h_{s}}^{\prime} \pi^{\prime}\left(g^{-1}\right)=\pi^{\prime}(g) \varepsilon_{g^{-1}}^{\prime} \pi^{\prime}\left(g^{-1}\right) \varepsilon_{g h_{1}}^{\prime} \ldots \varepsilon_{g h_{s}}^{\prime} \\
& =\varepsilon_{g}^{\prime} \varepsilon_{g h_{1}}^{\prime} \ldots \varepsilon_{g h_{s}}^{\prime}=\alpha_{g}\left(1_{g^{-1}} 1_{h_{1}} \ldots 1_{h_{s}}\right) .
\end{aligned}
$$

Consequently, the partial actions $\alpha^{\pi^{\prime}}$ and $\alpha$ coincide. On the other hand, the fact that $K^{n}$ is generated by the $1_{g}$ 's implies by Proposition 6.7 that the partial actions $\alpha$ and $\alpha^{\pi_{\alpha}}$ are equivalent.

It remains to observe that the partial representations $\pi^{\prime}$ and $\pi_{\alpha}$ are equivalent. Since $\alpha^{\pi^{\prime}}=\alpha$, we have by the first part of the proof the isomorphism $\phi_{\pi^{\prime}}: K^{n} \rtimes_{\alpha}$ $G \rightarrow M_{n}(K H)$, given by $\sum_{g \in G} a_{g} \delta_{g} \mapsto \sum_{g \in G} a_{g} \pi^{\prime}(g)\left(a_{g} \in \mathcal{D}_{g}\right)$. We see that $\phi_{\pi^{\prime}}\left(\pi_{\alpha}(g)\right)=\phi_{\pi^{\prime}}\left(1_{g} \delta_{g}\right)=\phi_{\pi^{\prime}}\left(\varepsilon_{g}^{\prime} \delta_{g}\right)=\varepsilon_{g}^{\prime} \pi^{\prime}(g)=\pi^{\prime}(g)$ for all $g \in G$. This yields the equivalence of $\pi^{\prime}$ and $\pi_{\alpha}$.

Corollary 6.12. For each elementary partial representation $\pi: G \rightarrow M_{n}(K H)$ the map $\phi_{\pi}: K^{n} \rtimes_{\alpha^{\pi}} G \rightarrow M_{n}(K H)$ given by $\sum_{g \in G} a_{g} \delta_{g} \mapsto \sum_{g \in G} a_{g} \pi(g)$ is an isomorphism.

Corollary 6.13. The partial action $\alpha^{\pi}$ which corresponds to an elementary partial representation $\pi: G \rightarrow M_{n}(K H)$ acts transitively on the minimal idempotents of $\operatorname{diag}(K, \ldots, K)$, i.e., for every $1 \leq i, j \leq n$ there exists an element $g \in G$ such that $e_{i, i}(1) \in \mathcal{D}_{g^{-1}}, e_{j, j}(1) \in \mathcal{D}_{g}$ and $\alpha_{g}\left(e_{i, i}(1)\right)=e_{j, j}(1)$.

Proof. For arbitrary fixed $1 \leq i, j \leq n$ there exists $g \in G$ with $g A_{i}=A_{j}$. Since $g^{-1} \in A_{i}$ and $\varepsilon_{g^{-1}}=\sum_{A_{k} \ni g^{-1}} e_{k k}(1)$, we see that $e_{i, i}(1) \in \varepsilon_{g^{-1}} \operatorname{diag}(K, \ldots, K)=$ $\mathcal{D}_{g^{-1}}$. By (91) and the equality $\prod_{f \in A_{i}} \varepsilon_{f}=e_{i i}(1)$ we have

$$
\begin{aligned}
& \alpha_{g}^{\pi}\left(e_{i, i}(1)\right)=\alpha_{g}^{\pi}\left(\prod_{f \in A_{i}} \varepsilon_{f}\right)=\pi(g) \prod_{f \in A_{i}} \varepsilon_{f} \pi\left(g^{-1}\right)=\prod_{f \in A_{i}} \varepsilon_{g f} \pi(g) \pi\left(g^{-1}\right) \\
& =\left(\prod_{f \in A_{j}} \varepsilon_{f}\right) \varepsilon_{g}=\prod_{f \in A_{j}} \varepsilon_{f}=e_{j, j}(1),
\end{aligned}
$$

as desired.

We see that the full matrix algebra $M_{n}(K H)$ can be viewed as a crossed product $K^{n} \rtimes_{\alpha} G$ in many ways. In particular, we have the following.

Corollary 6.14. For each positive integer $n$ and an arbitrary group $G$ of order $n+1$ there is a partial action $\alpha$ of $G$ on $K^{n}$ such that $M_{n}(K) \cong K^{n} \rtimes_{\alpha} G$.

Proof. Pick an element $1 \neq g \in G$ and take $A=G \backslash\{g\}$. Then $S t(A)=1$ and $A$ determines an elementary partial representation $\pi: G \rightarrow M_{n}(K)$, which gives rise to the isomorphism $\phi_{\pi}: K^{n} \rtimes_{\alpha^{\pi}} G \rightarrow M_{n}(K)$.

A crossed product structure on $M_{n}(K H)$ gives a grading of this $K$-algebra by $G$. It is easily seen that this grading is elementary. More precisely, we recall that a grading on the $K$-algebra $M_{n}(K)$ by a group $G$ is called elementary if each elementary matrix $e_{i, j}(1)$ is homogeneous. The elementary gradings are essential for the description of gradings on matrix algebras (see [3]). It is known that each elementary grading on $M_{n}(K)$ by a group $G$ is determined by an $n$ tuple $\left(g_{1}=1, g_{2}, \ldots, g_{n}\right)$ of not necessarily distinct elements of $G$ in such a way 
that the homogeneous degree $\operatorname{deg}\left(e_{i, j}(1)\right)$ of $e_{i, j}(1)$ is $g_{i}{ }^{-1} g_{j}$. Conversely, in this manner each $n$-tuple $\left(g_{1}=1, g_{2}, \ldots, g_{n}\right)$ determines an elementary grading on $M_{n}(K)$. It turns out that if in $\left(g_{1}=1, g_{2}, \ldots, g_{n}\right)$ the $g_{i}$ 's are pairwise distinct and $\operatorname{St}\left(\left\{g_{1}, g_{2}, \ldots, g_{n}\right\}\right)=1$, then the corresponding elementary grading of $M_{n}(K)$ necessarily comes from a crossed product structure $K^{n} \rtimes_{\alpha^{\pi}} G \cong M_{n}(K)$. More generally, we say that a grading of the $K$-algebra $M_{n}(K H)$ by a group $G$ is elementary if for each $h \in H, i, j \in\{1, \ldots, n\}$ the elementary matrix $e_{i, j}(h)$ is homogeneous. Given a subset $1 \in A$ of $G$ with $S t(A)=H$, one defines an elementary grading of $M_{n}(K H)$ by the equality $\operatorname{deg}\left(e_{i, j}(h)\right)=g_{i}{ }^{-1} h g_{j}(h \in H, i, j \in\{1, \ldots, n\})$, where $A=H g_{1} \cup H g_{2} \cup \ldots \cup H g_{n}, g_{1}=1$ and $H g_{i} \neq H g_{j}$ for $1 \leq i \neq j \leq n$. Changing the $g_{i}$ 's to $g_{i}{ }^{-1}$ 's in the definition of the elementary partial representations, we easily obtain the following.

Corollary 6.15. For the elementary grading of $M_{n}(K H)$ by a group $G$, determined by a subset $A \subseteq G$ with $A \ni 1, H=S t(A)$, and for the elementary partial representation $\pi: G \rightarrow M_{n}(K H)$, constructed from $A_{1}=A, A_{2}=g_{2}{ }^{-1} A, \ldots, A_{n}=g_{n}{ }^{-1} A$, the map $\phi_{\pi}: K^{n} \rtimes_{\alpha^{\pi}} G \rightarrow M_{n}(K H)$ is an isomorphism of graded $K$-algebras.

\section{ACKNOWLEDGMENTS}

While this work was carried out the first author visited the Federal University of Santa Catarina (Brazil) several times in 2001-2003. He expresses his appreciation to the Department of Mathematics of that university for its warm hospitality and financial support during the visits.

\section{REFERENCES}

[1] F. Abadie, Sobre ações parcias, fibrados de Fell e grupóides, PhD Thesis, Universidade de São Paulo, 1999.

[2] F. Abadie, Enveloping Actions and Takai Duality for Partial Actions, J. Funct. Anal. 197 (2003), 14-67. MR 2004c:46130

[3] Yu. Bahturin, S. K. Sehgal, M. V. Zaicev, Group gradings on associative algebras, J. Algebra, 241 (2001), 677-698. MR 2002h:16067

[4] J. Cuntz, W. Krieger, A Class of $C^{*}$-Algebras and Topological Markov Chains, Inventiones Math. 56 (1980), 251-268. MR 82f:46073a

[5] M. Dokuchaev, R. Exel, P. Piccione, Partial representations and partial group algebras, J. Algebra, 226 (2000), 505-532. MR 2001m:16034

[6] M. Dokuchaev, N. Zhukavets, On finite degree partial representations of groups, J. Algebra, 274 (2004), 309-334.

[7] M. Dokuchaev, N. Zhukavets, On irreducible partial representations of groups, Comptes Rendus Math. Rep. Acad. Sci. Canada, 24 (2002), 85-90.

[8] R. Exel, Circle actions on $C^{*}$-algebras, partial automorphisms and generalized PimsnerVoiculescu exact sequences, J. Funct. Anal. 122 (1994), 361-401. MR 95g:46122

[9] R. Exel, Twisted partial actions: a classification of regular $C^{*}$-algebraic bundles, Proc. London Math. Soc. 74 (1997), 417-443. MR 98d:46075

[10] R. Exel, Partial Actions of Groups and Actions of Semigroups, Proc. Am. Math. Soc. 126 (1998), 3481-3494. MR 99b:46102

[11] R. Exel, Amenability for Fell Bundles, J. Reine Angew. Math. 492 (1997), 41-73. MR 99a:46131

[12] R. Exel, M. Laca, Cuntz-Krieger Algebras for Infinite Matrices, J. Reine Angew. Math. 512 (1999), 119-172. MR 2000i:46064

[13] J. M. G. Fell, P. S. Doran, Representations of *-Algebras, Locally Compact Groups and Banach *-Algebraic Bundles I, Academic Press, 1988, Pure and Applied Math. 125. MR 90c:46001 
[14] P. A. Fillmore, A User's Guide to Operator Algebras, Willey - Interscience, 1996. MR 97i:46094

[15] K. McClanahan, K-theory for partial crossed products by discrete groups, J. Funct. Anal. 130 (1995), 77-117. MR 96i:46083

[16] D. S. Passman, The Algebraic Structure of Group Rings, Interscience, New York, 1977. MR 81d:16001

[17] J. C. Quigg, I. Raeburn, Characterizations of Crossed Products by Partial Actions, J. Operator Theory 37 (1997), 311-340. MR 99a:46121]

[18] L. H. Rowen, Ring theory - Student edition, Academic Press, 1991. MR 94e:16001

[19] S. K. Sehgal, Units in Integral Group Rings, Longman Scientific \& Technical Press, Harlow, 1993. MR 94m:16039

Departamento de Matemática, Universidade de São Paulo, Brazil

E-mail address: dokucha@ime.usp.br

Departamento de Matemática, Universidade Federal de Santa Catarina, Brazil

E-mail address: exel@mtm.ufsc.br

URL: http://www.mtm.ufsc.br/ ${ }^{\sim}$ exel 